\title{
POINT-LIKE PERTURBED FRACTIONAL LAPLACIANS THROUGH SHRINKING POTENTIALS OF FINITE RANGE
}

\author{
ALESSANDRO MICHELANGELI AND RAFFAELE SCANDONE
}

\begin{abstract}
We construct the rank-one, singular (point-like) perturbations of the $d$-dimensional fractional Laplacian in the physically meaningful normresolvent limit of fractional Schrödinger operators with regular potentials centred around the perturbation point and shrinking to a delta-like shape. We analyse both possible regimes, the resonance-driven and the resonance-independent limit, depending on the power of the fractional Laplacian and the spatial dimension. To this aim, we also qualify the notion of zero-energy resonance for Schrödinger operators formed by a fractional Laplacian and a regular potential.
\end{abstract}

\section{INTRODUCTION AND BACKGROUND}

In the last decade an amount of studies focused, in particular in application to the context of fractional quantum mechanics, on linear Schrödinger equations governed by the linear operator

$$
(-\Delta)^{s / 2}+\text { singular perturbation at } x_{0}
$$

for some fixed point $x_{0} \in \mathbb{R}^{d}$ and some $s>0$, that is, Schrödinger equations for a singular perturbation of a fractional power of the Laplacian [15, 17, 6, 13, 19, 21, 9, 16, 18.

Motivated by that, in a recent work in collaboration with A. Ottolini 14 we set up the systematic construction and classification of all the self-adjoint realisations in $L^{2}\left(\mathbb{R}^{d}\right)$ of the operators of the form (1.1) through a natural 'restriction-extension' procedure: first one restricts the operator $(-\Delta)^{s / 2}$ (initially defined, e.g., as a Fourier multiplier) to smooth functions vanishing in neighbourhoods of $x_{0}$, and then one builds all the operator extensions of such restriction that are self-adjoint on $L^{2}\left(\mathbb{R}^{d}\right)$.

This approach is surely satisfactory from the point of view of the interpretation of the output operator, which by construction is to be regarded as a point-like perturbation of the fractional Laplacian through an interaction supported only at $x_{0}$, say, " $(-\Delta)^{s / 2}+\delta\left(x-x_{0}\right)$ ". However, it obfuscates an amount of physical meaning, since it does not provide information, as the intuition would make one expect instead, on how the actual singular perturbation (1.1) is approximatively realised as a genuine pseudo-differential operator $(-\Delta)^{s / 2}+V\left(x-x_{0}\right)$ with a regular potential $V$ centred around $x=0$, with sufficiently short range and strong magnitude.

For the non-fractional Laplacian $-\Delta$ in $L^{2}\left(\mathbb{R}^{d}\right)$, the realisation of a singular perturbation at $x_{0} \in \mathbb{R}^{d}$ by means of approximating Schrödinger operators $-\Delta+V_{\varepsilon}$ with regular potentials $V_{\varepsilon}$ spiking up and shrinking around $x_{0}$ at a spatial scale $\varepsilon^{-1}$ in the limit $\varepsilon \downarrow 0$ is known since long for dimension $d=1$ [4],$d=2$ [1], and $d=3$ [2] (we also refer to [3, 5] for a comprehensive overview), that is, all the dimensions in which non-trivial singular perturbations exist.

Date: April 4, 2018.

Key words and phrases. Fractional Laplacian. Singular perturbations of differential operators. Schrödinger operators with shrinking potentials. Resolvent limits. Zero-energy resonance. 
The analogous question for the fractional Laplacian $(-\Delta)^{s / 2}$ was unanswered so far, and we solve it in the present work.

Not only is it topical in view of the above-mentioned recent mainstream in the literature of fractional Schrödinger equations with singular perturbation, but also it rises up the conceptually new issue of how a local potential $V_{\varepsilon}$ can be suitably rescaled so as to produce the desired perturbation of the non-local operator $(-\Delta)^{s / 2}$.

Let us first of all reconsider what emerges from the construction that, as mentioned, was recently given in 14.

For $s>0$ and $d \in \mathbb{N}$, the restriction $\left.(-\Delta)^{s / 2}\right|_{C_{0}^{\infty}\left(\mathbb{R}^{d} \backslash\{0\}\right)}$ is a positive symmetric operator on the Hilbert space $L^{2}\left(\mathbb{R}^{d}\right)$, hence with equal deficiency indices, and for short we shall just speak of the deficiency index. The number

$$
\mathcal{J}(s, d):=\text { deficiency index of }\left.(-\Delta)^{s / 2}\right|_{C_{0}^{\infty}\left(\mathbb{R}^{d} \backslash\{0\}\right)}
$$

is finite, and is zero or a strictly positive integer depending on $s$ and $d$, according to the rule

$$
\begin{aligned}
s \in I_{n}^{(d)} & :=\left\{\begin{array}{cc}
\left(0, \frac{d}{2}\right] & n=0 \\
\left(\frac{d}{2}+n-1, \frac{d}{2}+n\right] & n=1,2, \ldots
\end{array}\right. \\
& \Rightarrow \mathcal{J}(s, d)=\left(\begin{array}{c}
d+n-1 \\
d
\end{array}\right) .
\end{aligned}
$$

In the non-fractional case $s=2$ this yields the familiar values $\mathcal{J}(2,1)=2$, $\mathcal{J}(2,2)=1, \mathcal{J}(2,3)=1$, and $\mathcal{J}(2, d)=0$ for $d \geqslant 4$.

As well known, $\mathcal{J}(s, d)$ quantifies the infinite multiplicity of self-adjoint extensions of $\left.(-\Delta)^{s / 2}\right|_{C_{0}^{\infty}\left(\mathbb{R}^{d} \backslash\{0\}\right)}$ in $L^{2}\left(\mathbb{R}^{d}\right)$. By means of standard methods of the Krel̆n-Višik-Birman theory [8] one sees that the domain of each extension is formed by functions that are canonically decomposed into a regular $H^{s}$-component and a more singular component, the latter belonging to the $\mathcal{J}(s, d)$-dimensional kernel of $\left(\left.(-\Delta)^{s / 2}\right|_{C_{0}^{\infty}\left(\mathbb{R}^{d} \backslash\{0\}\right)}\right)^{*}+\lambda \mathbb{1}$ for some arbitrarily chosen $\lambda>0$, and satisfy an amount of 'boundary' (or 'contact') conditions between the evaluation at $x=0$ of the regular part or of some if its derivatives and the coefficients of the leading singularities of the singular part as $x \rightarrow 0$. Each set of boundary conditions identifies uniquely an extension.

For concreteness of the presentation, in this work we consider the self-adjoint extensions of $\left.(-\Delta)^{s / 2}\right|_{C_{0}^{\infty}\left(\mathbb{R}^{d} \backslash\{0\}\right)}$ in the case of deficiency index 1 only, and for simplicity we omit further the explicit discussion of the 'endpoint' values of $s$, namely the largest possible value, at given $d$, compatible with $\mathcal{J}(s, d)=1$. As expressed by (1.2), this amounts to analysing the regime $s \in\left(\frac{1}{2}, \frac{3}{2}\right)$ in $d=1$, $s \in(1,2)$ in $d=2, s \in\left(\frac{3}{2}, \frac{5}{2}\right)$ in $d=3$, etc., where the considered intervals are the non-endpoint values of $s$, the endpoint value being $s=\frac{d}{2}+1$. We shall refer to such cases as the ' $\mathcal{J}=1$ scenario'. For this scenario we then discuss how to realise the corresponding extensions in the limit of Schrödinger operators with fractional Laplacian and shrinking potentials, say, $(-\Delta)^{s / 2}+V_{\varepsilon}$ as $\varepsilon \downarrow 0$.

In fact, it will be evident from our discussion that the behaviour and the control of the limit $\varepsilon \downarrow 0$ in the $\mathcal{J}=1$ scenario is technically the very same irrespectively of the dimension, and therefore we will pick up a concrete value of $d$ for the explicit computations, modulo the dichotomy that we now describe.

When $\mathcal{J}(s, d)=1$, and $s \neq \frac{d}{2}+1$, the space where the above-mentioned singular components run over, namely $\operatorname{ker}\left(\left(\left.(-\Delta)^{s / 2}\right|_{C_{0}^{\infty}\left(\mathbb{R}^{d} \backslash\{0\}\right)}\right)^{*}+\lambda \mathbb{1}\right)$, is the onedimensional space spanned by the Green function $\mathrm{G}_{s, \lambda}$ of the fractional Laplacian, defined by

$$
\left((-\Delta)^{s / 2}+\lambda\right) \mathrm{G}_{s, \lambda}=\delta(x)
$$


for higher deficiency index the kernel is spanned by $\mathrm{G}_{s, \lambda}$ and other non- $H^{s}$ functions. Now, depending on $d$ and $s$, the Green function $\mathrm{G}_{s, 0}$ may be singular or regular at $x=0$ : when $s<d, \mathrm{G}_{s, 0}$ has a singularity $\sim|x|^{-(d-s)}$, it has a logarithmic singularity when $s=d$, and it is continuous at $x=0$ when $s>d$. Omitting the transition case, which does not alter the conceptual scheme of the present discussion and could be easily recovered with analogous arguments to those that we shall use when $s<d$, we thus distinguish two possibilities in the $\mathcal{J}=1$ scenario, that we call

- Locally singular, or ResonanCe-Driven CASE: $s<d$,

- LOCAlly Regular, or ResonanCE-INDEPEndent CASE: $s>d$.

We shall explain in a moment the meaning of the 'resonance' jargon: it has to do with how the limit of shrinking potentials must be organised in order to reach a self-adjoint extension of $\left.(-\Delta)^{s / 2}\right|_{C_{0}^{\infty}\left(\mathbb{R}^{d} \backslash\{0\}\right)}$ in one case or in the other. Also, let us remark that an analogous dichotomy occurs when the deficiency index of $\left.(-\Delta)^{s / 2}\right|_{C_{0}^{\infty}\left(\mathbb{R}^{d} \backslash\{0\}\right)}$ is larger than 1: the singular (non- $H^{s}$ ) component of the elements in the domain of the considered self-adjoint extension may or may not display a local singularity as $x \rightarrow 0$.

In view of the above alternative, we make the following presentational choice. Since in all dimensions $d$ but $d=1$ the interval $s \in\left(\frac{d}{2}, \frac{d}{2}+1\right)$ corresponding to deficiency index 1 lies strictly below the transition value $s=d$ that separates the locally regular from the locally singular regime, as a representative of any such value of $d$ for concreteness we choose $d=3$ : the discussion on the limit of shrinking potentials would then be immediately exportable to any other $d \geqslant 2$. Next to that, we also discuss the case $d=1$, where instead the interval $s \in(1,2)$ corresponding to deficiency index 1 contains the transition value $s=1$.

As mentioned above, self-adjoint extensions of $\left.(-\Delta)^{s / 2}\right|_{C_{0}^{\infty}\left(\mathbb{R}^{d} \backslash\{0\}\right)}$ in the locally regular and the locally singular case differ both in the type of non-regularity of the functions in their domain at $x=0$, and, as we shall show in this work, in the type of approximating Schrödinger operators $(-\Delta)^{s / 2}+V_{\varepsilon}$, meaning, in the scaling chosen for $V_{\varepsilon}$ and, most importantly, in the spectral requirements.

Extensions in the locally regular case can be reached as $\varepsilon \downarrow 0$ through suitably rescaled versions $V_{\varepsilon}$ of a given potential $V$ with no further prescription on $V$ but those technical assumptions ensuring that the limit itself is well-posed. Instead, extensions in the locally singular case can only be reached if the unscaled operator $(-\Delta)^{s / 2}+V$ admits a zero-energy resonance, a spectral behaviour at the bottom of its essential spectrum which we shall define in due time and roughly speaking amounts to the existence of a suitably decaying, non square-integrable, $L_{\text {loc }}^{2}$-solution $f$ to $\left((-\Delta)^{s / 2}+V\right) f=0$. In a sense that we shall make precise, this difference is due to the fact that a zero-energy resonance is needed in the approximating fractional Schrödinger operator in order to reproduce in the limit the locally singular behaviour in the domain of the considered self-adjoint extension.

In fact, the phenomenon we have just described is the generalisation for $(-\Delta)^{s / 2}$ of what is well known for $-\Delta$ (i.e., $s=2$ in the present notation). When $d=1$, the deficiency index of $\left.(-\Delta)\right|_{C_{0}^{\infty}(\mathbb{R} \backslash\{0\})}$ equals 2 and

$$
\operatorname{ker}\left(\left(\left.(-\Delta)\right|_{C_{0}^{\infty}(\mathbb{R} \backslash\{0\})}\right)^{*}+\lambda \mathbb{1}\right)=\operatorname{span}\left\{\frac{1}{\sqrt{\lambda}} e^{-\sqrt{\lambda}|x|},(\operatorname{sign} x) e^{-\sqrt{\lambda}|x|}\right\}
$$

(in particular, $\mathrm{G}_{2, \lambda}(x)=\sqrt{\frac{\pi}{2 \lambda}} e^{-\sqrt{\lambda}|x|}$ ), therefore the functions in the above space are less regular than $H^{2}(\mathbb{R})$ but not locally singular at $x=0$. The so-called $\delta$-type extensions, namely those in the domain of which the singular component is $e^{-\sqrt{\lambda}|x|}$, can indeed be realised as limits of $-\Delta+V_{\varepsilon}$ with no spectral requirement needed at energy zero for the unscaled $-\Delta+V$ [3, Chapt. I.3]. On the contrary, when $d=3$ 
the deficiency index of $\left.(-\Delta)\right|_{C_{0}^{\infty}\left(\mathbb{R}^{3} \backslash\{0\}\right)}$ equals 1 and

$$
\operatorname{ker}\left(\left(\left.(-\Delta)\right|_{C_{0}^{\infty}\left(\mathbb{R}^{3} \backslash\{0\}\right)}\right)^{*}+\lambda \mathbb{1}\right)=\operatorname{span}\left\{\mathrm{G}_{2, \lambda}\right\}, \quad \mathrm{G}_{2, \lambda}(x)=\frac{e^{-\sqrt{\lambda}|x|}}{4 \pi|x|},
$$

thus with a local singularity at $x=0$. The self-adjoint extensions of $\left.(-\Delta)\right|_{C_{0}^{\infty}\left(\mathbb{R}^{3} \backslash\{0\}\right)}$ can be realised as limits of $-\Delta+V_{\varepsilon}$ provided that $-\Delta+V$ is zero-energy resonant [3. Chapt. I.1]. In the former situation we are in the locally regular, resonantindependent case; in the latter we are in the locally singular, resonant-driven case.

The material of this work is organised as follows.

- In Section 2 we define the singular perturbations of the three-dimensional fractional Laplacian and we present the approximation scheme in terms of fractional Schrödinger operators with regular, shrinking potentials.

- In Section 3 we present the one-dimensional analogue, including the definition of the singular perturbations and the two distinct approximation schemes, for the resonance-driven and the resonance-independent cases.

- Section 4 contains the proof of the three-dimensional limit.

- Section 5 contains the proof of the one-dimensional limit in the resonancedriven case. From the technical point of view, the argument here is completely analogous to that of 4 , as the $3 \mathrm{D}$ case too is resonance-driven.

- Section 6 contains instead the proof of the one-dimensional limit in the resonance-independent case.

- In Section 7 we prove a technical result used in the main proofs, that is, the characterisation of the zero-energy resonant behaviour of the unscaled operator $(-\Delta)^{s / 2}+V$. Then, we discuss the occurrence of zero-energy resonances.

Let us conclude this Introduction with a few comments about our otherwise standard notation. For an operator $T$ on a Hilbert space, $\mathcal{D}(T)$ denotes its operator domain and, when $T$ is self-adjoint, $\mathcal{D}[T]$ denotes its form domain. We shall denote by $\mathbb{1}$, resp., by $\mathbb{D}$, the identity and the null operator on any of the considered Hilbert spaces. We shall indicate the Fourier transform by $\widehat{\phi}$ or $\mathcal{F} \phi$ with the convention $\widehat{\phi}(p)=(2 \pi)^{-\frac{d}{2}} \int_{\mathbb{R}^{d}} e^{-\mathrm{i} p x} \phi(x) \mathrm{d} x$. We shall write $A \lesssim B$ for $A \leqslant$ const. $B$ when the constant does not depend on the other relevant parameters or variables of both sides of the inequality; for $x \in \mathbb{R}^{d}$ we shall write $\langle x\rangle:=\left(1+x^{2}\right)^{\frac{1}{2}}$.

\section{Approximation SCheme in Dimension three}

In this Section we consider the singular perturbations of the three-dimensional fractional Laplacian and their approximation by means of fractional Schrödinger operators with shrinking potentials.

Let us start with the densely defined, closed, positive, symmetric operator

$$
\grave{\mathrm{k}}^{(s / 2)}:=\overline{(-\Delta)^{s / 2}\left\lceil C_{0}^{\infty}\left(\mathbb{R}^{3} \backslash\{0\}\right)\right.}, \quad s>0,
$$

with respect to the Hilbert space $L^{2}\left(\mathbb{R}^{3}\right)$. In 14 we presented the construction and classification of the self-adjoint extensions of $\mathrm{k}^{(s / 2)}$, which we recall here below.

Clearly, for small enough powers $s, \stackrel{\mathrm{k}}{(s / 2)}^{(s)}$ already self-adjoint, thus with no room for point-like singular perturbations, indeed [14, Lemma A.1]

$$
\mathcal{D}\left(\grave{\mathrm{k}}^{(s / 2)}\right)=H_{0}^{s}\left(\mathbb{R}^{3} \backslash\{0\}\right)=\overline{C_{0}^{\infty}\left(\mathbb{R}^{3} \backslash\{0\}\right)} \|_{H^{s}}=H^{s}\left(\mathbb{R}^{3}\right), \quad \text { if } s \in\left[0, \frac{3}{2}\right) .
$$


When $s$ increases, the domain of $\dot{\mathrm{k}}^{(s / 2)}$ is qualified by an increasing number of conditions for $H^{s}$-functions, namely [14, Lemma A.2]

$$
\begin{gathered}
\mathcal{D}\left(\mathrm{k}^{(s / 2)}\right)=\left\{\begin{array}{c}
f \in H^{s}\left(\mathbb{R}^{3}\right) \text { such that } \\
\int_{\mathbb{R}^{3}} p_{1}^{\gamma_{1}} p_{2}^{\gamma_{2}} p_{3}^{\gamma_{3}} \widehat{f}(p) \mathrm{d} p=0 \\
\gamma_{1}, \gamma_{2}, \gamma_{3} \in \mathbb{N}_{0}, \gamma_{1}+\gamma_{2}+\gamma_{3} \leqslant n-1
\end{array}\right\} \\
\text { if } s \in I_{n}^{(3)}:=\left(n+\frac{1}{2}, n+\frac{3}{2}\right), \quad n \in \mathbb{N} ;
\end{gathered}
$$

as a consequence, the adjoint of $\dot{k}^{(s / 2)}$ becomes strictly larger than $\dot{k}^{(s / 2)}$ itself, with an increasingly complicated structure of its domain that reflects the fact that for $s \in I_{n}^{(3)}$ the deficiency index of $\dot{\mathrm{k}}^{(s / 2)}$ equals $\left(\begin{array}{c}n+2 \\ 3\end{array}\right)$, and this in turn affects the structure of the family of its self-adjoint extensions.

In particular, in the regime $s \in I_{1}^{(3)}=\left(\frac{3}{2}, \frac{5}{2}\right)$ one has

$$
\mathcal{D}\left(\grave{\mathrm{k}}^{(s / 2)}\right)=\left\{f \in H^{s}\left(\mathbb{R}^{3}\right) \mid \int_{\mathbb{R}^{3}} \widehat{f}(p) \mathrm{d} p=0\right\} \quad \text { if } s \in\left(\frac{3}{2}, \frac{5}{2}\right)
$$

and $\mathfrak{k}^{(s / 2)}$ has deficiency index 1, which leaves room for a one-(real-)parameter family of self-adjoint extensions. In order to qualify them, for chosen $\lambda>0$ and $s \in \mathbb{R}$ let us denote the Green's function as

$$
\mathrm{G}_{s, \lambda}(x):=\frac{1}{(2 \pi)^{\frac{3}{2}}}\left(\frac{1}{|p|^{s}+\lambda}\right)^{\vee}(x), \quad x, p \in \mathbb{R}^{3} .
$$

By construction, distributionally.

$$
\left((-\Delta)^{s / 2}+\lambda\right) \mathrm{G}_{s, \lambda}=\delta(x)
$$

Observe that $\mathrm{G}_{s, \lambda}$ has a local singularity $|x|^{-(3-s)}$, more precisely [14, Sec. 3],

$$
\mathrm{G}_{s, \lambda}(x)=\frac{\Lambda_{s}}{|x|^{(3-s)}}+\mathrm{J}_{s, \lambda}(x)
$$

with

$$
\begin{aligned}
\Lambda_{s} & :=\frac{\Gamma\left(\frac{3-s}{2}\right)}{(2 \pi)^{\frac{3}{2}} 2^{s-\frac{3}{2}} \Gamma\left(\frac{s}{2}\right)} \\
\mathrm{J}_{s, \lambda} & :=-\frac{\lambda}{(2 \pi)^{\frac{3}{2}}}\left(\frac{1}{|p|^{s}\left(|p|^{s}+\lambda\right)}\right)^{\vee} \in C_{\infty}\left(\mathbb{R}^{3}\right) .
\end{aligned}
$$

The following construction/classification Theorem was established in [14].

Theorem 2.1. Let $s \in\left(\frac{3}{2}, \frac{5}{2}\right)$.

(i) The self-adjoint extensions in $L^{2}\left(\mathbb{R}^{3}\right)$ of the operator $\dot{\mathrm{k}}^{(s / 2)}$ form the family $\left(\mathrm{k}_{\alpha}^{(s / 2)}\right)_{\alpha \in \mathbb{R} \cup\{\infty\}}$, where $\mathrm{k}_{\infty}^{(s / 2)}$ is its Friedrichs extension, namely the selfadjoint fractional Laplacian $(-\Delta)^{s / 2}$, and all other (proper) extensions are given, for arbitrary $\lambda>0$, by

$$
\begin{aligned}
\mathcal{D}\left(\mathrm{k}_{\alpha}^{(s / 2)}\right) & =\left\{g=F^{\lambda}+\frac{F^{\lambda}(0)}{\alpha-\frac{\lambda^{\frac{3}{s}}-1}{2 \pi s \sin \left(\frac{3 \pi}{s}\right)}} \mathrm{G}_{s, \lambda} \mid F^{\lambda} \in H^{s}\left(\mathbb{R}^{3}\right)\right\} \\
\left(\mathrm{k}_{\alpha}^{(s / 2)}+\lambda\right) g & =\left((-\Delta)^{s / 2}+\lambda\right) F^{\lambda} .
\end{aligned}
$$


(ii) For each $\alpha \in \mathbb{R}$ the quadratic form of the extension $\mathrm{k}_{\alpha}^{(s / 2)}$ is given by

$$
\begin{gathered}
\mathcal{D}\left[\mathrm{k}_{\alpha}^{(s / 2)}\right]=H^{\frac{s}{2}}\left(\mathbb{R}^{3}\right) \dot{+} \operatorname{span}\left\{\mathrm{G}_{s, \lambda}\right\} \\
\mathrm{k}_{\alpha}^{(s / 2)}\left[F^{\lambda}+\kappa_{\lambda} \mathrm{G}_{s, \lambda}\right]=\left\||\nabla|^{s} F^{\lambda}\right\|_{L^{2}\left(\mathbb{R}^{3}\right)}^{2}-\lambda\left\|F^{\lambda}+\kappa_{\lambda} \mathrm{G}_{s, \lambda}\right\|_{L^{2}\left(\mathbb{R}^{3}\right)}^{2} \\
+\lambda\left\|F^{\lambda}\right\|_{L^{2}\left(\mathbb{R}^{3}\right)}^{2}+\left(\alpha-\frac{\lambda^{\frac{3}{s}-1}}{2 \pi s \sin \left(\frac{3 \pi}{s}\right)}\right)\left|\kappa_{\lambda}\right|^{2}
\end{gathered}
$$

for arbitrary $\lambda>0$.

(iii) The resolvent of $\mathrm{k}_{\alpha}^{(s / 2)}$ is given by

$$
\begin{aligned}
\left(\mathrm{k}_{\alpha}^{(s / 2)}+\lambda \mathbb{1}\right)^{-1}= & \left((-\Delta)^{s / 2}+\lambda \mathbb{1}\right)^{-1} \\
& +\left(\alpha-\frac{\lambda^{\frac{3}{s}-1}}{2 \pi s \sin \left(\frac{3 \pi}{s}\right)}\right)^{-1}\left|\mathrm{G}_{s, \lambda}\right\rangle\left\langle\mathrm{G}_{s, \lambda}\right|
\end{aligned}
$$

for arbitrary $\lambda>0$

(iv) Each extension is semi-bounded from below, and

$$
\begin{aligned}
\sigma_{\mathrm{ess}}\left(\mathrm{k}_{\alpha}^{(s / 2)}\right) & =\sigma_{\mathrm{ac}}\left(\mathrm{k}^{(s / 2)}\right)=[0,+\infty), \\
\sigma_{\mathrm{disc}}\left(\mathrm{k}_{\alpha}^{(s / 2)}\right) & =\left\{\begin{array}{cl}
\emptyset & \text { if } \alpha \geqslant 0 \\
\left\{E_{\alpha}^{(s)}\right\} & \text { if } \alpha<0,
\end{array}\right.
\end{aligned}
$$

where the eigenvalue $E_{\alpha}^{(s)}$ is non-degenerate and is given by

$$
E_{\alpha}^{(s)}=-\left(2 \pi|\alpha| s \sin \left(-\frac{3 \pi}{s}\right)\right)^{\frac{s}{3-s}},
$$

the (non-normalised) eigenfunction being $\mathrm{G}_{s, \lambda=\left|E_{\alpha}^{(s)}\right|}$.

Our goal now is to qualify each of the extensions given by Theorem 2.1 as suitable limits of approximating fractional Schrödinger operators with finite range potentials.

It is convenient to introduce the class $\mathcal{R}_{s, d}, d \in \mathbb{N}, s \in\left(\frac{d}{2}, d\right)$, of measurable functions $V: \mathbb{R}^{d} \rightarrow \mathbb{C}$ such that

$$
\iint_{\mathbb{R}^{d} \times \mathbb{R}^{d}} \mathrm{~d} x \mathrm{~d} y \frac{|V(x)||V(y)|}{|x-y|^{2(d-s)}}=:\|V\|_{\mathcal{R}_{s, d}}^{2}<+\infty .
$$

$\mathcal{R}_{2,3}$ is the well-known Rollnick class on $\mathbb{R}^{3}$. Clearly, $\mathcal{R}_{s, d} \supset C_{0}^{\infty}\left(\mathbb{R}^{d}\right)$.

For each $s \in\left(\frac{3}{2}, \frac{5}{2}\right)$ we make the following assumption.

\section{Assumption $\left(\mathbf{I}_{s}\right)$.}

(i) $V: \mathbb{R}^{3} \rightarrow \mathbb{R}$ is a measurable function in $L^{1}\left(\mathbb{R}^{3},\langle x\rangle^{2 s-3} \mathrm{~d} x\right) \cap \mathcal{R}_{s, 3}$.

(ii) $\eta: \mathbb{R} \rightarrow \mathbb{R}^{+}$is a continuous function satisfying $\eta(0)=\eta(1)=1$ and

$$
\eta(\varepsilon)=1+\eta_{s} \varepsilon^{3-s}+o\left(\varepsilon^{3-s}\right) \quad \text { as } \varepsilon \downarrow 0
$$

for some $\eta_{s} \in \mathbb{R}$ that we call the strength of the distortion factor $\eta$.

For given $V$ and $\eta$ satisfying Assumption $\left(\mathrm{I}_{s}\right)$, let us set

$$
h_{\varepsilon}^{(s / 2)}:=(-\Delta)^{s / 2}+V_{\varepsilon}, \quad V_{\varepsilon}(x):=\frac{\eta(\varepsilon)}{\varepsilon^{s}} V\left(\frac{x}{\varepsilon}\right), \quad \varepsilon>0 .
$$

For every $\varepsilon>0$ the operator $h_{\varepsilon}^{(s / 2)}$, defined as a form sum, is self-adjoint on $L^{2}\left(\mathbb{R}^{3}\right)$ and $\sigma_{\text {ess }}\left(h_{\varepsilon}^{(s / 2)}\right)=[0,+\infty)($ Lemma 4.1(iii)).

The spectral properties of the unscaled operator $(-\Delta)^{s / 2}+V$ at the bottom of the essential spectrum are crucial for the limit $\varepsilon \downarrow 0$ in $h_{\varepsilon}^{(s / 2)}$. In the next Theorem we qualify the zero-energy behaviour of $(-\Delta)^{s / 2}+V$.

Theorem 2.2. Let $s \in\left(\frac{3}{2}, \frac{5}{2}\right), V \in L^{1}\left(\mathbb{R}^{3},\langle x\rangle^{2 s-3} \mathrm{~d} x\right) \cap \mathcal{R}_{s, 3}$, real-valued. Let $v:=|V|^{\frac{1}{2}}$ and $u:=|V|^{\frac{1}{2}} \operatorname{sign}(V)$.

(i) The operator $u(-\Delta)^{-\frac{s}{2}} v$ is compact on $L^{2}\left(\mathbb{R}^{3}\right)$. 
Assume in addition that

$$
u(-\Delta)^{-\frac{s}{2}} v \phi=-\phi \quad \text { for some } \phi \in L^{2}\left(\mathbb{R}^{3}\right) \backslash\{0\}
$$

and define

$$
\psi:=(-\Delta)^{-\frac{s}{2}} v \phi
$$

Then:

(ii) $\psi \in L_{\mathrm{loc}}^{2}\left(\mathbb{R}^{3}\right)$ and $\left((-\Delta)^{s / 2}+V\right) \psi=0$ in the sense of distributions,

(iii) $\langle v, \phi\rangle_{L^{2}}=-\int_{\mathbb{R}^{3}} \mathrm{~d} x V(x) \psi(x)$,

(iv) $\psi \in L^{2}\left(\mathbb{R}^{3}\right) \Leftrightarrow\langle v, \phi\rangle_{L^{2}}=0$, in which case $\psi \in \mathcal{D}\left((-\Delta)^{s / 2}+V\right)$.

When a $L^{2}$-function $\phi$ exists that satisfies (2.17) and the corresponding function $\psi$ defined by (2.18) belongs to $L_{\text {loc }}^{2}\left(\mathbb{R}^{3}\right) \backslash L^{2}\left(\mathbb{R}^{3}\right)$ we say that $(-\Delta)^{s / 2}+V$ is zeroenergy resonant and that $\psi$ is a zero-energy resonance for $(-\Delta)^{s / 2}+V$. If for the zero-energy resonant operator $(-\Delta)^{s / 2}+V$ the eigenvalue -1 of $u(-\Delta)^{-\frac{s}{2}} v$ is non-degenerate, then we say that the resonance is simple. Of course, if $\psi \in L^{2}\left(\mathbb{R}^{3}\right)$, then $\psi$ is an eigenfunction of $(-\Delta)^{s / 2}+V$ with eigenvalue zero.

We shall prove Theorem 2.2 in Section 7 together with a discussion of the occurrence of a zero-energy resonance for $(-\Delta)^{s / 2}+V$ (Proposition 7.1).

Let us now formulate our main result for dimension three. It is the control of the approximation, in the norm resolvent sense, of the singular perturbation operator $k_{\alpha}^{(s / 2)}$ by means of Schrödinger operators with the $\frac{s}{2}$-th fractional Laplacian and shrinking potentials $V_{\varepsilon}$ around the origin. We shall prove it in Section 4 .

Theorem 2.3. Let $s \in\left(\frac{3}{2}, \frac{5}{2}\right)$. Given a potential $V$ and a distortion factor $\eta$ with strength $\eta_{s}$ satisfying Assumption $\left(\mathrm{I}_{s}\right)$, for every $\varepsilon>0$ let $h_{\varepsilon}^{(s / 2)}=(-\Delta)^{s / 2}+V_{\varepsilon}$ be the corresponding self-adjoint Schrödinger operator defined in (2.16) with the $\frac{s}{2}$-th fractional Laplacian and the shrinking potential $V_{\varepsilon}$.

(i) If $(-\Delta)^{s / 2}+V$ is not zero-energy resonant, then $h_{\varepsilon}^{(s / 2)} \stackrel{\varepsilon \downarrow 0}{\longrightarrow}(-\Delta)^{s / 2}$ in the norm-resolvent sense on $L^{2}\left(\mathbb{R}^{3}\right)$.

(ii) If $(-\Delta)^{s / 2}+V$ admits a simple zero-energy resonance $\psi$, then for

$$
\alpha:=-\eta_{s}\left|\int_{\mathbb{R}^{3}} \mathrm{~d} x V(x) \psi(x)\right|^{-2}
$$

one has $h_{\varepsilon}^{(s / 2)} \stackrel{\varepsilon \downarrow 0}{\longrightarrow} \mathrm{k}_{\alpha}^{(s / 2)}$ in the norm-resolvent sense on $L^{2}\left(\mathbb{R}^{3}\right)$.

In view of the discussion we made in the introductory Section, the two possible alternatives in Theorem 2.3 are the manifestation of the locally singular, resonantdriven nature of the limit: the limit is well-posed for a generic class of potentials $V$, but it is non-trivial only if additionally $(-\Delta)^{s / 2}+V$ is zero-energy resonant.

By a simple scaling argument one sees that $(-\Delta)^{s / 2}+V_{\varepsilon}$ remains zero-energy resonant for any $\varepsilon>0$ if the scaling is 'purely geometric', namely with trivial distortion factor, $\eta(\varepsilon) \equiv 1$. In this case, the signature of the resonance is particularly transparent: as stated in Theorem 2.3 (ii), the limit $\varepsilon \downarrow 0$ with $\eta(\varepsilon) \equiv 1$ produces the extension parametrised by $\alpha=0$ and we see from Theorem 2.1(iv) that the negative eigenvalue of $\mathrm{k}_{\alpha}^{(s / 2)}$ when $\alpha<0$ converges to 0 as $\alpha \uparrow 0$, with the corresponding eigenfunction $\mathrm{G}_{s, \lambda=\left|E_{\alpha}^{(s)}\right|}$ converging pointwise to $\mathrm{G}_{s, 0}(x)=\frac{\Lambda_{s}}{|x|^{(3-s)}}$ (see (2.7)-(2.8) and (2.14) above); the $L_{\text {loc }}^{2} \backslash L^{2}$-function $\mathrm{G}_{s, 0}$ can be actually regarded as a zero-energy resonance for $\mathrm{k}_{\alpha=0}^{(s / 2)}$ (the local square-integrability following from $\left.s \in\left(\frac{3}{2}, \frac{5}{2}\right)\right)$. 


\section{Approximation scheme in Dimension one}

In this Section we consider the singular perturbations of the one-dimensional fractional Laplacian and their approximation by means of fractional Schrödinger operators with shrinking potentials.

As for the $3 \mathrm{D}$ case, for $\lambda>0$ and $s \geqslant 0$, we set

$$
\mathrm{G}_{s, \lambda}(x):=\frac{1}{(2 \pi)^{\frac{1}{2}}}\left(\frac{1}{|p|^{s}+\lambda}\right)^{\vee}(x), \quad x, p \in \mathbb{R}
$$

(whence $\left((-\Delta)^{s / 2}+\lambda\right) \mathrm{G}_{s, \lambda}=\delta(x)$ distributionally), and

$$
\grave{k}^{(s / 2)}:=\overline{(-\Delta)^{s / 2} \uparrow C_{0}^{\infty}(\mathbb{R} \backslash\{0\})}
$$

as an operator closure with respect to the Hilbert space $L^{2}(\mathbb{R}) . \stackrel{\circ}{k}^{(s / 2)}$ has deficiency index $n \in \mathbb{N}$ when $s \in I_{n}^{(1)}:=\left(n-\frac{1}{2}, n+\frac{1}{2}\right)$ (see (1.2) above), and in the case of deficiency index 1 one has

$$
\mathcal{D}\left(\mathfrak{k}^{(s / 2)}\right)=\left\{f \in H^{s}(\mathbb{R}) \mid \int_{\mathbb{R}} \widehat{f}(p) \mathrm{d} p=0\right\}, \quad s \in\left(\frac{1}{2}, \frac{3}{2}\right)
$$

(which can be seen by means of a completely analogous argument to that of 14, Appendix A]). The corresponding one-(real-)parameter family of self-adjoint extensions is given by the one-dimensional analogous of Theorem 2.1 14 .

Theorem 3.1. Let $s \in\left(\frac{1}{2}, \frac{3}{2}\right)$ and

$$
\Theta(s, \lambda):=\left\{\begin{array}{cl}
\left(\lambda^{1-\frac{1}{s}} s \sin \left(\frac{\pi}{s}\right)\right)^{-1} & s \neq 1 \\
-\frac{1}{\pi} \ln \lambda & s=1,
\end{array} \quad \lambda>0 .\right.
$$

(i) The self-adjoint extensions in $L^{2}(\mathbb{R})$ of the operator $\dot{\mathrm{k}}^{(s / 2)}$ form the family $\left(\mathrm{k}_{\alpha}^{(s / 2)}\right)_{\alpha \in \mathbb{R} \cup\{\infty\}}$, where $\mathrm{k}_{\infty}^{(s / 2)}$ is its Friedrichs extension, namely the selfadjoint fractional Laplacian $(-\Delta)^{s / 2}$, and all other (proper) extensions are given, for arbitrary $\lambda>0$, by

$$
\begin{aligned}
\mathcal{D}\left(\mathrm{k}_{\alpha}^{(s / 2)}\right) & =\left\{\begin{array}{c}
g=F^{\lambda}+\frac{F^{\lambda}(0)}{\alpha-\Theta(s, \lambda)} \mathrm{G}_{s, \lambda} \\
F^{\lambda} \in H^{s}(\mathbb{R})
\end{array}\right\} \\
\left(\mathrm{k}_{\alpha}^{(s / 2)}+\lambda\right) g & =\left((-\Delta)^{s / 2}+\lambda\right) F^{\lambda} .
\end{aligned}
$$

(ii) For each $\alpha \in \mathbb{R}$ the quadratic form of the extension $\mathrm{k}_{\alpha}^{(s / 2)}$ is given by

$$
\begin{aligned}
& \mathcal{D}\left[\mathrm{k}_{\alpha}^{(s / 2)}\right]=H^{\frac{s}{2}}(\mathbb{R}) \dot{+} \operatorname{span}\left\{\mathrm{G}_{s, \lambda}\right\} \\
& \mathrm{k}_{\alpha}^{(s / 2)}\left[F^{\lambda}+\kappa_{\lambda} \mathrm{G}_{s, \lambda}\right]=\left\||\nabla|^{s} F^{\lambda}\right\|_{L^{2}(\mathbb{R})}^{2}-\lambda\left\|F^{\lambda}+\kappa_{\lambda} \mathrm{G}_{s, \lambda}\right\|_{L^{2}(\mathbb{R})}^{2} \\
& +\lambda\left\|F^{\lambda}\right\|_{L^{2}(\mathbb{R})}^{2}+(\alpha-\Theta(s, \lambda))\left|\kappa_{\lambda}\right|^{2}
\end{aligned}
$$

for arbitrary $\lambda>0$.

(iii) The resolvent of $\mathrm{k}_{\alpha}^{(s / 2)}$ is given by

$$
\begin{aligned}
\left(\mathrm{k}_{\alpha}^{(s / 2)}+\lambda \mathbb{1}\right)^{-1}= & \left((-\Delta)^{s / 2}+\lambda \mathbb{1}\right)^{-1} \\
& +(\alpha-\Theta(s, \lambda))^{-1}\left|\mathrm{G}_{s, \lambda}\right\rangle\left\langle\mathrm{G}_{s, \lambda}\right|
\end{aligned}
$$

for arbitrary $\lambda>0$.

(iv) For each $\alpha \in \mathbb{R}$ the extension $\mathrm{k}_{\alpha}^{(s / 2)}$ is semi-bounded from below, and

$$
\sigma_{\mathrm{ess}}\left(\mathrm{k}_{\alpha}^{(s / 2)}\right)=\sigma_{\mathrm{ac}}\left(\mathrm{k}_{\alpha}^{(s / 2)}\right)=[0,+\infty), \quad \sigma_{\mathrm{sc}}\left(\mathrm{k}_{\alpha}^{(s / 2)}\right)=\emptyset,
$$




$$
\sigma_{\mathrm{disc}}\left(\mathrm{k}_{\alpha}^{(s / 2)}\right)=\left\{\begin{array}{cl}
\emptyset & \text { if } s \neq 1,(s-1) \alpha \leqslant 0 \\
\left\{-E_{\alpha}^{(s)}\right\} & \text { if } s \neq 1,(s-1) \alpha>0 \\
\left\{-E_{\alpha}^{(1)}\right\} & \text { if } s=1,
\end{array}\right.
$$

where the eigenvalue $-E_{\alpha}^{(s)}$ is non-degenerate and is given by

$$
E_{\alpha}^{(s)}=\left\{\begin{array}{cl}
\left(\alpha s \sin \left(\frac{\pi}{s}\right)\right)^{\frac{s}{1-s}} & s \neq 1 \\
e^{-\pi \alpha} & s=1,
\end{array}\right.
$$

the (non-normalised) eigenfunction being $\mathrm{G}_{s, \lambda=\left|E_{\alpha}^{(s)}\right|}$.

Our goal is to qualify each of the extensions given by Theorem 3.1 as suitable limits of approximating fractional Schrödinger operators with finite range potentials. Unlike the 3D setting, here the regime $s \in\left(\frac{1}{2}, \frac{3}{2}\right)$ is separated by the transition value $s=1$, below which we are in the locally singular case for the Green function (3.1), and above which we are in the locally regular case, in the terminology of Section 1 This will result in different assumptions on the approximating potentials and different schemes for the resolvent limit.

We therefore proceed by splitting our discussion into the two above-mentioned cases.

\subsection{Locally singular, resonance-driven case.}

This is the regime $s \in\left(\frac{1}{2}, 1\right)$. The Green function $\mathrm{G}_{s, \lambda}$ has a local singularity,

$$
\mathrm{G}_{s, \lambda}(x)=\frac{\Lambda_{s}}{|x|^{(1-s)}}+\mathrm{J}_{s, \lambda}(x)
$$

with

$$
\begin{aligned}
\Lambda_{s} & :=\frac{\Gamma\left(\frac{1-s}{2}\right)}{(2 \pi)^{\frac{1}{2}} 2^{s-\frac{1}{2}} \Gamma\left(\frac{s}{2}\right)} \\
\mathrm{J}_{s, \lambda} & :=-\frac{\lambda}{(2 \pi)^{\frac{1}{2}}}\left(\frac{1}{|p|^{s}\left(|p|^{s}+\lambda\right)}\right)^{\vee} \in C_{\infty}(\mathbb{R}) .
\end{aligned}
$$

We make the following assumption (the class $R_{s, d}$ was introduced in (2.15)).

Assumption $\left(\mathbf{I}_{s}^{-}\right) . s \in\left(\frac{1}{2}, 1\right)$ and moreover:

(i) $V: \mathbb{R} \rightarrow \mathbb{R}$ is a measurable function in $L^{1}\left(\mathbb{R},\langle x\rangle^{2 s-1} \mathrm{~d} x\right) \cap \mathcal{R}_{s, 1}$;

(ii) $\eta: \mathbb{R} \rightarrow \mathbb{R}^{+}$is a continuous function satisfying $\eta(0)=\eta(1)=1$ and

$$
\eta(\varepsilon)=1+\eta_{s} \varepsilon^{1-s}+o\left(\varepsilon^{1-s}\right) \quad \text { as } \varepsilon \downarrow 0
$$

for some $\eta_{s} \in \mathbb{R}$ that we call the strength of the distortion factor $\eta$.

For given $V$ and $\eta$ satisfying Assumption $\left(\mathrm{I}_{s}^{-}\right)$, let us set

$$
h_{\varepsilon}^{(s / 2)}:=(-\Delta)^{s / 2}+V_{\varepsilon}, \quad V_{\varepsilon}(x):=\frac{\eta(\varepsilon)}{\varepsilon^{s}} V\left(\frac{x}{\varepsilon}\right), \quad \varepsilon>0 .
$$

For every $\varepsilon>0$ the operator $h_{\varepsilon}^{(s / 2)}$, defined as a form sum, is self-adjoint on $L^{2}\left(\mathbb{R}^{3}\right)$ and $\sigma_{\text {ess }}\left(h_{\varepsilon}^{(s / 2)}\right)=[0,+\infty)($ Lemma $5.2($ iii $))$.

The zero-energy spectral behaviour of $(-\Delta)^{s / 2}+V$, which is crucial for the limit $\varepsilon \downarrow 0$ in $h_{\varepsilon}^{(s / 2)}$, is described as follows, in analogy with Theorem 2.2

Theorem 3.2. Let $s \in\left(\frac{1}{2}, 1\right), V \in L^{1}\left(\mathbb{R},\langle x\rangle^{2 s-1} \mathrm{~d} x\right) \cap \mathcal{R}_{s, 1}$, real-valued. Let $v:=|V|^{\frac{1}{2}}$ and $u:=|V|^{\frac{1}{2}} \operatorname{sign}(V)$.

(i) The operator $u(-\Delta)^{-\frac{s}{2}} v$ is compact on $L^{2}(\mathbb{R})$. 
Assume in addition that

$$
u(-\Delta)^{-\frac{s}{2}} v \phi=-\phi \quad \text { for some } \phi \in L^{2}(\mathbb{R}) \backslash\{0\}
$$

and define

$$
\psi:=(-\Delta)^{-\frac{s}{2}} v \phi
$$

Then:

(ii) $\psi \in L_{\mathrm{loc}}^{2}(\mathbb{R})$ and $\left((-\Delta)^{s / 2}+V\right) \psi=0$ in the sense of distributions,

(iii) $\langle v, \phi\rangle_{L^{2}}=-\int_{\mathbb{R}} \mathrm{d} x V(x) \psi(x)$,

(iv) $\psi \in L^{2}(\mathbb{R}) \Leftrightarrow\langle v, \phi\rangle_{L^{2}}=0$, in which case $\psi \in \mathcal{D}\left((-\Delta)^{s / 2}+V\right)$.

We defer to Section 7 the proof of Theorem 3.2 and a discussion of the occurrence of a zero-energy resonance for $(-\Delta)^{s / 2}+V$ (Proposition [7.2). With the same terminology of Section 2, $(-\Delta)^{s / 2}+V$ is zero-energy resonant and that $\psi$ is a zero-energy resonance for $(-\Delta)^{s / 2}+V$ when there exists a non-zero $L^{2}$-function $\phi$ satisfying (3.15) and the corresponding function $\psi$ defined by (3.16) belongs to $L_{\text {loc }}^{2}(\mathbb{R}) \backslash L^{2}\left(\mathbb{R}^{)}\right.$. If, for the zero-energy resonant operator $(-\Delta)^{s / 2}+V$, the eigenvalue -1 of $u(-\Delta)^{-\frac{s}{2}} v$ is non-degenerate, then the resonance is simple.

Here below is our first main result in dimension one, relative to the resonancedriven regime.

Theorem 3.3. Let $s \in\left(\frac{1}{2}, 1\right)$. Given a potential $V$ and a distortion factor $\eta$ with strength $\eta_{s}$ satisfying Assumption $\left(\mathrm{I}_{s}^{-}\right)$, for every $\varepsilon>0$ let $h_{\varepsilon}^{(s / 2)}=(-\Delta)^{s / 2}+V_{\varepsilon}$ be the corresponding self-adjoint Schrödinger operator defined in (3.14) with the $\frac{s}{2}-t h$ fractional Laplacian and the shrinking potential $V_{\varepsilon}$.

(i) If $(-\Delta)^{s / 2}+V$ is not zero-energy resonant, then $h_{\varepsilon}^{(s / 2)} \stackrel{\varepsilon \downarrow 0}{\longrightarrow}(-\Delta)^{s / 2}$ in the norm-resolvent sense on $L^{2}(\mathbb{R})$.

(ii) If $(-\Delta)^{s / 2}+V$ admits a simple zero-energy resonance $\psi$, then for

$$
\alpha:=-\eta_{s}\left|\int_{\mathbb{R}} \mathrm{d} x V(x) \psi(x)\right|^{-2}
$$

one has $h_{\varepsilon}^{(s / 2)} \stackrel{\varepsilon \downarrow 0}{\longrightarrow} \mathrm{k}_{\alpha}^{(s / 2)}$ in the norm-resolvent sense on $L^{2}(\mathbb{R})$.

We shall prove Theorem 3.3 in Section 5

The alternative in Theorem 3.3 is completely analogous to that of Theorem 2.3 . due to the the locally singular, resonant-driven nature of both limits: only for zero-energy resonant operators $(-\Delta)^{s / 2}+V$ is the limit non-trivial.

The signature of the resonance is particularly transparent in the absence of distortion factor: when $\eta(\varepsilon) \equiv 1$ by scaling one sees that $(-\Delta)^{s / 2}+V_{\varepsilon}$ remains zero-energy resonant for any $\varepsilon>0$, and we may regard the limit operator $\mathrm{k}_{\alpha=0}^{(s / 2)}$ too as zero-energy resonant, for the negative eigenvalue of $k_{\alpha}^{(s / 2)}$ when $|\alpha| \neq 0$ vanishes as $|\alpha| \rightarrow 0$ and the corresponding eigenfunctions becomes (proportional to) the $L_{\text {loc }}^{2} \backslash L^{2}$-function $|x|^{-(1-s)}$ (see (3.11) above).

\subsection{Locally regular, resonance-independent case.}

This is the regime $s \in\left(1, \frac{3}{2}\right)$. In contrast with the resonance-driven regime, no spectral requirement is now needed on the unscaled fractional operator $(-\Delta)^{s / 2}+V$ and the scaling in $V_{\varepsilon}$ is independent of $s$. Thus, we make the following assumption.

\section{Assumption $\left(\mathbf{I}_{s}^{+}\right)$.}

(i) $V: \mathbb{R} \rightarrow \mathbb{R}$ is a measurable function in $L^{1}(\mathbb{R})$.

(ii) $\eta: \mathbb{R}^{+} \rightarrow \mathbb{R}^{+}$is a smooth function satisfying $\eta(1)=1$. 
Correspondingly, we set

$$
h_{\varepsilon}^{(s / 2)}:=(-\Delta)^{s / 2}+V_{\varepsilon}, \quad V_{\varepsilon}(x):=\frac{\eta(\varepsilon)}{\varepsilon} V\left(\frac{x}{\varepsilon}\right), \quad \varepsilon>0 .
$$

For every $\varepsilon>0$ the operator $h_{\varepsilon}^{(s / 2)}$, defined as a form sum, is self-adjoint on $L^{2}\left(\mathbb{R}^{3}\right)$ and $\sigma_{\text {ess }}\left(h_{\varepsilon}^{(s / 2)}\right)=[0,+\infty)($ Lemma $6.1($ iii $))$.

Here below is our second main result in dimension one, which, as opposite to Theorem 3.3, takes the following form.

Theorem 3.4. Let $s \in\left(1, \frac{3}{2}\right)$. For every $\varepsilon>0$ let $h_{\varepsilon}^{(s / 2)}=(-\Delta)^{s / 2}+V_{\varepsilon}$ be defined according to Assumption (Is) and (3.17). Then $h_{\varepsilon}^{(s / 2)} \stackrel{\varepsilon \downarrow 0}{\longrightarrow} \mathrm{k}_{\alpha}^{(s / 2)}$ in the norm-resolvent sense on $L^{2}(\mathbb{R})$, where

$$
\alpha:=-\left(\eta(0) \int_{\mathbb{R}} \mathrm{d} x V(x)\right)^{-1} .
$$

We shall prove Theorem 3.4 in Section 6 .

\section{Convergence of the 3D limit}

The goal of this Section is to prove Theorem 2.3

Let us start with qualifying the following useful operator-theoretic properties.

Lemma 4.1. Let $V: \mathbb{R}^{3} \rightarrow \mathbb{R}$ belong to $L^{1}\left(\mathbb{R}^{3}\right) \cap \mathcal{R}_{s, 3}$ for some $s \in\left(\frac{3}{2}, 3\right)$. Then:

(i) for every $\lambda \geqslant 0,|V|^{\frac{1}{2}}\left((-\Delta)^{\frac{s}{2}}+\lambda \mathbb{1}\right)^{-1}|V|^{\frac{1}{2}}$ is a Hilbert-Schmidt operator on $L^{2}\left(\mathbb{R}^{3}\right)$

(ii) $|V|^{\frac{1}{2}} \ll(-\Delta)^{\frac{s}{4}}$ in the sense of infinitesimally bounded operators;

(iii) the operator $(-\Delta)^{\frac{s}{2}}+V$ defined as a form sum is self-adjoint and $\sigma_{\mathrm{ess}}\left((-\Delta)^{\frac{s}{2}}+\right.$ $V)=[0,+\infty)$.

Proof. (i) $|V|^{\frac{1}{2}}\left((-\Delta)^{\frac{s}{2}}+\lambda \mathbb{1}\right)^{-1}|V|^{\frac{1}{2}}$ acts as an integral operator with kernel

$$
\mathcal{K}_{s, \lambda}(x, y):=|V(x)|^{\frac{1}{2}} \mathrm{G}_{s, \lambda}(x-y)|V(y)|^{\frac{1}{2}},
$$

and its Hilbert-Schmidt norm is estimated as

$$
\begin{aligned}
& \left\||V|^{\frac{1}{2}}\left((-\Delta)^{\frac{s}{2}}+\lambda \mathbb{1}\right)^{-1}|V|^{\frac{1}{2}}\right\|_{\mathrm{H} . \mathrm{S} .}^{2}=\iint_{\mathbb{R}^{3} \times \mathbb{R}^{3}} \mathrm{~d} x \mathrm{~d} y\left|\mathcal{K}_{s, \lambda}(x, y)\right|^{2} \\
& \quad \leqslant 2 \Lambda_{s}^{2} \iint_{\mathbb{R}^{3} \times \mathbb{R}^{3}} \mathrm{~d} x \mathrm{~d} y \frac{|V(x)||V(y)|}{|x|^{2(3-s)}}+2\left\|\mathrm{~J}_{s, \lambda}\right\|_{L^{\infty}}^{2} \iint_{\mathbb{R}^{3} \times \mathbb{R}^{3}} \mathrm{~d} x \mathrm{~d} y|V(x)||V(y)| \\
& \quad \leqslant 2 \Lambda_{s}^{2}\|V\|_{\mathcal{R}_{s, 3}}^{2}+2\left\|\mathrm{~J}_{s, \lambda}\right\|_{L^{\infty}}^{2}\|V\|_{L^{1}}^{2}<+\infty
\end{aligned}
$$

having used (2.7)-(2.8) in the second step.

(ii) The map $\lambda \mapsto|V|^{\frac{1}{2}}\left((-\Delta)^{\frac{s}{2}}+\lambda \mathbb{1}\right)^{-1}|V|^{\frac{1}{2}}$ is continuous from $(0,+\infty)$ to the space of Hilbert-Schmidt operators, and by dominated convergence

$$
\lim _{\lambda \rightarrow+\infty} \iint_{\mathbb{R}^{3} \times \mathbb{R}^{3}} \mathrm{~d} x \mathrm{~d} y|V(x)|\left|\mathrm{G}_{s, \lambda}(x-y)\right|^{2}|V(y)|=0 .
$$

Therefore, for arbitrary $\varepsilon>0$ it is possible to find $\lambda_{\varepsilon}>0$ large enough such that

$$
\begin{aligned}
\varepsilon & \geqslant\left\||V|^{\frac{1}{2}}\left((-\Delta)^{\frac{s}{2}}+\lambda_{\varepsilon} \mathbb{1}\right)^{-1}|V|^{\frac{1}{2}}\right\|_{\text {H.S. }}^{2} \\
& =\left\|\left((-\Delta)^{\frac{s}{2}}+\lambda_{\varepsilon} \mathbb{1}\right)^{-\frac{1}{2}}|V|\left((-\Delta)^{\frac{s}{2}}+\lambda_{\varepsilon} \mathbb{1}\right)^{-\frac{1}{2}}\right\|_{\text {H.S. }}^{2} \\
& \geqslant\left\|\left((-\Delta)^{\frac{s}{2}}+\lambda_{\varepsilon} \mathbb{1}\right)^{-\frac{1}{2}}|V|\left((-\Delta)^{\frac{s}{2}}+\lambda_{\varepsilon} \mathbb{1}\right)^{-\frac{1}{2}}\right\|_{\mathrm{op}}^{2},
\end{aligned}
$$

which implies, for some $b_{\varepsilon}>0$,

$$
\left|\langle\varphi, V \varphi\rangle_{L^{2}}\right| \leqslant \varepsilon\left\langle\varphi,(-\Delta)^{\frac{s}{2}} \varphi\right\rangle_{L^{2}}+b_{\varepsilon}\|\varphi\|_{L^{2}}^{2} \quad \forall \varphi \in \mathcal{D}\left[(-\Delta)^{\frac{s}{2}}\right]=H^{\frac{s}{2}}\left(\mathbb{R}^{3}\right),
$$


and hence $|V|^{\frac{1}{2}} \ll(-\Delta)^{\frac{s}{4}}$.

(iii) The statement follows at once from (ii).

For chosen $s \in\left(\frac{3}{2}, \frac{5}{2}\right), \varepsilon>0$, and $V$ and $\eta$ satisfying Assumption $\left(\mathrm{I}_{s}\right)$, let us recall from (2.16) that $V_{\varepsilon}(x)=\frac{\eta(\varepsilon)}{\varepsilon^{s}} V\left(\frac{x}{\varepsilon}\right)$ and let us define

$$
\begin{aligned}
v(x) & :=|V(x)|^{\frac{1}{2}}, \quad u(x):=|V(x)|^{\frac{1}{2}} \operatorname{sign}(V(x)), \\
v_{\varepsilon}(x) & :=\left|V_{\varepsilon}(x)\right|^{\frac{1}{2}}, \quad u_{\varepsilon}(x):=\left|V_{\varepsilon}(x)\right|^{\frac{1}{2}} \operatorname{sign}\left(V_{\varepsilon}(x)\right) .
\end{aligned}
$$

Thus,

$$
v_{\varepsilon}(x)=\frac{\sqrt{\eta(\varepsilon)}}{\varepsilon^{s / 2}} v\left(\frac{x}{\varepsilon}\right), \quad u_{\varepsilon}(x)=\frac{\sqrt{\eta(\varepsilon)}}{\varepsilon^{s / 2}} u\left(\frac{x}{\varepsilon}\right), \quad v_{\varepsilon} u_{\varepsilon}=V_{\varepsilon} .
$$

The Hamiltonian $h_{\varepsilon}^{(s / 2)}=(-\Delta)^{s / 2}+V_{\varepsilon}$ defined in (2.16) as a form sum is selfadjoint on $L^{2}\left(\mathbb{R}^{3}\right)$, as guaranteed by Lemma 4.1(iii). An expression for its resolvent that is convenient in the present context is the Konno-Kuroda identity [12. One has the following.

Lemma 4.2. Let $V: \mathbb{R}^{3} \rightarrow \mathbb{R}$ belong to $L^{1}\left(\mathbb{R}^{3}\right) \cap \mathcal{R}_{s, 3}$ for some $s \in\left(\frac{3}{2}, \frac{5}{2}\right)$. Then

$$
\begin{aligned}
& \left(h_{\varepsilon}^{(s / 2)}+\lambda \mathbb{1}\right)^{-1}=\left((-\Delta)^{s / 2}+\lambda \mathbb{1}\right)^{-1}- \\
& -\left((-\Delta)^{s / 2}+\lambda \mathbb{1}\right)^{-1} v_{\varepsilon}\left(\mathbb{1}+u_{\varepsilon}\left((-\Delta)^{s / 2}+\lambda \mathbb{1}\right)^{-1} v_{\varepsilon}\right)^{-1} u_{\varepsilon}\left((-\Delta)^{s / 2}+\lambda \mathbb{1}\right)^{-1}
\end{aligned}
$$

for every $\varepsilon>0$ and every $-\lambda<0$ in the resolvent set of $h_{\varepsilon}^{(s / 2)}$, as an identity between bounded operators on $L^{2}\left(\mathbb{R}^{3}\right)$.

Proof. The statement is precisely the application of the Konno-Kuroda resolvent identity, for which we follow the formulation presented in [3, Theorem B.1(b)], to the operator $(-\Delta)^{s / 2}+v_{\varepsilon} u_{\varepsilon}$. For the validity of such identity two conditions are needed: the compactness of $u_{\varepsilon}\left((-\Delta)^{s / 2}+\lambda \mathbb{1}\right)^{-1} v_{\varepsilon}$ and the infinitesimal bound $|V|^{\frac{1}{2}} \ll(-\Delta)^{\frac{s}{4}}$. Both conditions are guaranteed by Lemma 4.1

Observe that the invertibility of $\mathbb{1}+u_{\varepsilon}\left((-\Delta)^{s / 2}+\lambda \mathbb{1}\right)^{-1} v_{\varepsilon}$ (with bounded inverse) is part of the statement of the Konno-Kuroda formula (4.3).

It is convenient to manipulate the identity (4.3) further so as to isolate terms in the r.h.s. which are easily controllable in the limit $\varepsilon \downarrow 0$. To this aim, let us introduce for each $\varepsilon>0$ the unitary scaling operator $U_{\varepsilon}: L^{2}\left(\mathbb{R}^{3}\right) \rightarrow L^{2}\left(\mathbb{R}^{3}\right)$ defined by

$$
\left(U_{\varepsilon} f\right)(x):=\frac{1}{\varepsilon^{3 / 2}} f\left(\frac{x}{\varepsilon}\right) .
$$

Its adjoint clearly acts as $\left(U_{\varepsilon}^{*} f\right)(x)=\varepsilon^{3 / 2} f(\varepsilon x)$. $U_{\varepsilon}$ induces the scaling transformations

$$
\begin{aligned}
U_{\varepsilon}^{*} v_{\varepsilon} U_{\varepsilon} & =\frac{\sqrt{\eta(\varepsilon)}}{\varepsilon^{s / 2}} v, \quad U_{\varepsilon}^{*} u_{\varepsilon} U_{\varepsilon}=\frac{\sqrt{\eta(\varepsilon)}}{\varepsilon^{s / 2}} u, \\
U_{\varepsilon}^{*}\left((-\Delta)^{s / 2}+\lambda \mathbb{1}\right)^{-1} U_{\varepsilon} & =\varepsilon^{s}\left((-\Delta)^{s / 2}+\lambda \varepsilon^{s} \mathbb{1}\right)^{-1},
\end{aligned}
$$

whose proof is straightforward.

Let us also introduce, for each $\varepsilon>0$ and for each $\mu>0$ such that $-\mu^{s}$ belongs to the resolvent set of $h_{\varepsilon}^{(s / 2)}$, the operators

$$
\begin{aligned}
& A_{\varepsilon}^{(s)}:=\varepsilon^{-\frac{3-s}{2}}\left((-\Delta)^{s / 2}+\mu^{s} \mathbb{1}\right)^{-1}(\eta(\varepsilon))^{-\frac{1}{2}} v_{\varepsilon} U_{\varepsilon} \\
& B_{\varepsilon}^{(s)}:=\eta(\varepsilon) u\left((-\Delta)^{s / 2}+(\mu \varepsilon)^{s} \mathbb{1}\right)^{-1} v \\
& C_{\varepsilon}^{(s)}:=U_{\varepsilon}^{*} u_{\varepsilon}(\eta(\varepsilon))^{-\frac{1}{2}}\left((-\Delta)^{s / 2}+\mu^{s} \mathbb{1}\right)^{-1} \varepsilon^{-\frac{3-s}{2}} .
\end{aligned}
$$


We shall see in a moment (Lemma 4.4) that $A_{\varepsilon}^{(s)}, B_{\varepsilon}^{(s)}$, and $C_{\varepsilon}^{(s)}$ are HilbertSchmidt operators on $L^{2}\left(\mathbb{R}^{3}\right)$. Most importantly for our purposes, the resolvent of $h_{\varepsilon}^{(s / 2)}$ takes the following convenient form.

Lemma 4.3. Under the present assumptions,

$$
\left(h_{\varepsilon}^{(s / 2)}+\mu^{s} \mathbb{1}\right)^{-1}=\left((-\Delta)^{s / 2}+\mu^{s} \mathbb{1}\right)^{-1}-A_{\varepsilon}^{(s)} \varepsilon^{3-s} \eta(\varepsilon)\left(\mathbb{1}+B_{\varepsilon}^{(s)}\right)^{-1} C_{\varepsilon}^{(s)}
$$

for every $\varepsilon>0$ and every $\mu>0$ such that $-\mu^{s}$ belongs to the resolvent set of $h_{\varepsilon}^{(s / 2)}$.

Proof. In formula (4.3) we set $\lambda=\mu^{s}$ and we insert $\mathbb{1}=U_{\varepsilon} U_{\varepsilon}^{*}$ in the second summand of the r.h.s. right after $\left((-\Delta)^{s / 2}+\lambda \mathbb{1}\right)^{-1} v_{\varepsilon}$. We then commute $U_{\varepsilon}^{*}$ all the way through by means of the scaling transformations (4.5): this way, we reproduce the product $A_{\varepsilon}^{(s)} \varepsilon^{3-s} \eta(\varepsilon)\left(\mathbb{1}+B_{\varepsilon}^{(s)}\right)^{-1} C_{\varepsilon}^{(s)}$.

The limit $\varepsilon \downarrow 0$ can be monitored explicitly for $A_{\varepsilon}^{(s)}, B_{\varepsilon}^{(s)}$, and $C_{\varepsilon}^{(s)}$.

Lemma 4.4. For every $\varepsilon>0, A_{\varepsilon}^{(s)}, B_{\varepsilon}^{(s)}$, and $C_{\varepsilon}^{(s)}$ are Hilbert-Schmidt operators on $L^{2}\left(\mathbb{R}^{3}\right)$ with limit

$$
\begin{aligned}
& \lim _{\varepsilon \downarrow 0} A_{\varepsilon}^{(s)}=\left|\mathrm{G}_{s, \mu^{s}}\right\rangle\langle v| \\
& \lim _{\varepsilon \downarrow 0} B_{\varepsilon}^{(s)}=B_{0}^{(s)}=u(-\Delta)^{-\frac{s}{2}} v \\
& \lim _{\varepsilon \downarrow 0} C_{\varepsilon}^{(s)}=|u\rangle\left\langle\mathrm{G}_{s, \mu^{s}}\right|
\end{aligned}
$$

in the Hilbert-Schmidt operator norm.

Proof. By construction, see (4.2), (4.4), and (4.6) above,

$$
\begin{aligned}
\left(A_{\varepsilon}^{(s)} f\right)(x) & =\varepsilon^{-\frac{3-s}{2}} \varepsilon^{-\frac{s}{2}} \varepsilon^{-\frac{3}{2}} \int_{\mathbb{R}^{3}} \mathrm{G}_{s, \mu^{s}}(x-y) v\left(\frac{y}{\varepsilon}\right) f\left(\frac{y}{\varepsilon}\right) \mathrm{d} y \\
& =\int_{\mathbb{R}^{3}} \mathrm{G}_{s, \mu^{s}}(x-\varepsilon y) v(y) f(y) \mathrm{d} y \quad \forall f \in L^{2}\left(\mathbb{R}^{3}\right),
\end{aligned}
$$

that is, $A_{\varepsilon}^{(s)}$ acts as an integral operator with kernel $\mathrm{G}_{s, \mu^{s}}(x-\varepsilon y) v(y)$. The latter is clearly a function in $L^{2}\left(\mathbb{R}^{3} \times \mathbb{R}^{3}, \mathrm{~d} x \mathrm{~d} y\right)$ uniformly in $\varepsilon$, and dominated convergence implies

$$
\left\|A_{\varepsilon}^{(s)}\right\|_{\mathrm{H} . \mathrm{S} .}^{2}=\iint_{\mathbb{R}^{3} \times \mathbb{R}^{3}} \mathrm{~d} x \mathrm{~d} y\left|\mathrm{G}_{s, \mu^{s}}(x-\varepsilon y) v(y)\right|^{2} \stackrel{\varepsilon \downarrow 0}{\longrightarrow}\left\|\mathrm{G}_{s, \mu^{s}}\right\|_{L^{2}}^{2}\|V\|_{L^{1}}
$$

as well as

$$
\begin{aligned}
\left\langle g, A_{\varepsilon}^{(s)} f\right\rangle_{L^{2}} & =\iint_{\mathbb{R}^{3} \times \mathbb{R}^{3}} \mathrm{~d} x \mathrm{~d} y \overline{g(x)} \mathrm{G}_{s, \mu^{s}}(x-\varepsilon y) v(y) f(y) \\
& \stackrel{\varepsilon \downarrow 0}{\longrightarrow}\left\langle g, \mathrm{G}_{s, \mu^{s}}\right\rangle_{L^{2}}\langle v, f\rangle_{L^{2}} \quad \forall f, g \in C_{0}^{\infty}\left(\mathbb{R}^{3}\right) .
\end{aligned}
$$

As a consequence, as $\varepsilon \downarrow 0, A_{\varepsilon}^{(s)} \rightarrow\left|\mathrm{G}_{s, \mu^{s}}\right\rangle\langle v|$ weakly in the operator topology, and the Hilbert-Schmidt norm of $A_{\varepsilon}^{(s)}$ converges to the Hilbert-Schmidt norm of its limit. By a well-known feature of compact operators [20, Theorem 2.21], the combination of these two properties implies that $A_{\varepsilon}^{(s)} \rightarrow\left|\mathrm{G}_{s, \mu^{s}}\right\rangle\langle v|$ in the HilbertSchmidt topology. This proves (4.8).

The discussion for $C_{\varepsilon}^{(s)}$ is completely analogous: its integral kernel is $u(x) \mathrm{G}_{s, \mu^{s}}(\varepsilon x-$ $y$ ) and (4.10) is proved by the very same type of argument.

Concerning $B_{\varepsilon}^{(s)}$, its integral kernel is $\eta(\varepsilon) u(x) \mathrm{G}_{s,(\mu \varepsilon)^{s}}(x-y) v(y)$ and the integral kernel of $B_{0}^{(s)}$ is $u(x) \mathrm{G}_{s, 0}(x-y) v(y)$ : owing to Lemma 4.1(i) both operators are Hilbert Schmidt, and moreover by dominated convergence $B_{\varepsilon}^{(s)} \rightarrow B_{0}^{(s)}$ weakly in 


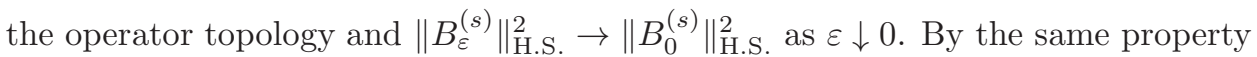
20. Theorem 2.21] the limit (4.9) then holds in the Hilbert-Schmidt norm.

It is evident from (4.7) that, in order for the limits (4.8)-4.10) above to qualify the behaviour of the resolvent of $h_{\varepsilon}^{(s / 2)}$ as $\varepsilon \downarrow 0$, one needs additional information on the possible failure of invertibility in $L^{2}\left(\mathbb{R}^{3}\right)$ of the operator $\mathbb{1}+B_{0}^{(s)}$. By the Fredholm alternative, since $B_{0}^{(s)}$ is compact, $\left(\mathbb{1}+B_{0}^{(s)}\right)^{-1}$ exists everywhere defined and bounded, in which case (4.7) implies at once $\left(h_{\varepsilon}^{(s / 2)}+\mu^{s} \mathbb{1}\right)^{-1} \rightarrow\left((-\Delta)^{s / 2}+\right.$ $\left.\mu^{s} \mathbb{1}\right)^{-1}$ as $\varepsilon \downarrow 0$, unless $B_{0}^{(s)}$ admits an eigenvalue -1 .

Let us then assume that the latter circumstance does occurs, namely condition (2.17) of Theorem 2.2 More precisely, we make the following assumption.

Assumption $\left(\mathbf{I I}_{s}\right)$. Assumption $\left(\mathrm{I}_{s}\right)$ holds. $B_{0}^{(s)}$ has eigenvalue -1 , which is non-degenerate. $\phi \in L^{2}\left(\mathbb{R}^{3}\right)$ is a non-zero function such that $B_{0}^{(s)} \phi=-\phi$ and, in addition, $\langle\widetilde{\phi}, \phi\rangle_{L^{2}}=-1$, where $\widetilde{\phi}:=(\operatorname{sign} V) \phi$.

Since $\langle\widetilde{\phi}, \phi\rangle_{L^{2}}=-\left\langle(\operatorname{sign} V) \phi,(\operatorname{sign} V) v(-\Delta)^{-\frac{s}{2}} v \phi\right\rangle_{L^{2}}=-\left\|(-\Delta)^{-\frac{s}{4}} v \phi\right\|_{L^{2}}^{2}$, the normalisation $\langle\widetilde{\phi}, \phi\rangle_{L^{2}}=-1$ is always possible.

Under Assumption $\left(\mathrm{II}_{s}\right),\left(\mathbb{1}+B_{\varepsilon}^{(s)}\right)^{-1}$ becomes singular in the limit $\varepsilon \downarrow 0$, with a singularity that now competes with the vanishing factor $\varepsilon^{3-s}$ of (4.7). To resolve this competing effect, we need first an expansion of $B_{\varepsilon}^{(s)}$ around $\varepsilon=0$ to a further order, than the limit (4.9). This expansion holds irrespectively of Assumption $\left(\mathrm{II}_{s}\right)$.

Lemma 4.5. Let $s \in\left(\frac{3}{2}, \frac{5}{2}\right)$ and $\lambda>0$.

(i) For every $x \in \mathbb{R}^{3} \backslash\{0\}$

$$
\lim _{\lambda \downarrow 0} \frac{\mathrm{G}_{s, \lambda}(x)-\mathrm{G}_{s, 0}(x)}{\left(2 \pi s \sin \left(\frac{3 \pi}{s}\right)\right)^{-1} \lambda^{\frac{3}{s}-1}}=1 .
$$

(ii) In the norm operator topology one has

$$
\lim _{\varepsilon \downarrow 0} \frac{1}{(\mu \varepsilon)^{3-s}}\left(B_{\varepsilon}^{(s)}-B_{0}^{(s)}\right)=\frac{\eta_{s}}{\mu^{3-s}} B_{0}^{(s)}+\frac{1}{2 \pi s \sin \left(\frac{3 \pi}{s}\right)}|u\rangle\langle v| .
$$

Here $\mu>0$ is the constant chosen in the definition (4.6) of $B_{\varepsilon}^{(s)}$ and $\eta_{s} \in \mathbb{R}$ is the constant that is part of Assumption $\left(\mathrm{I}_{s}\right)$.

Proof. (i) From (2.5) we write

$$
\begin{aligned}
\frac{\mathrm{G}_{s, \lambda}(x)-\mathrm{G}_{s, 0}(x)}{\lambda^{\frac{3}{s}-1}} & =\frac{1}{\lambda^{\frac{3}{s}-1}(2 \pi)^{\frac{3}{2}}} \int_{\mathbb{R}^{3}} \mathrm{~d} p e^{\mathrm{i} x \cdot p} \frac{-\lambda}{(2 \pi)^{\frac{3}{2}}|p|^{s}\left(|p|^{s}+\lambda\right)} \\
& =-\frac{1}{(2 \pi)^{3}} \int_{\mathbb{R}^{3}} \mathrm{~d} p e^{\mathrm{i} \lambda^{1 / s} x \cdot p} \frac{1}{|p|^{s}\left(|p|^{s}+1\right)},
\end{aligned}
$$

whence

$$
\frac{\mathrm{G}_{s, \lambda}(x)-\mathrm{G}_{s, 0}(x)}{\lambda^{\frac{3}{s}-1}} \stackrel{\lambda \downarrow 0}{\longrightarrow}-\frac{1}{(2 \pi)^{3}} \int_{\mathbb{R}^{3}} \mathrm{~d} p \frac{1}{|p|^{s}\left(|p|^{s}+1\right)}=\frac{1}{2 \pi s \sin \left(\frac{3 \pi}{s}\right)}
$$

by dominated convergence, since $p \mapsto\left(|p|^{s}\left(|p|^{s}+1\right)\right)^{-1}$ is integrable when $s \in\left(\frac{3}{2}, 3\right)$.

(ii) The Hilbert-Schmidt operator

$$
\frac{1}{(\mu \varepsilon)^{3-s}}\left(B_{\varepsilon}^{(s)}-B_{0}^{(s)}\right)-\frac{\eta_{s}}{\mu^{3-s}} B_{0}^{(s)}-\frac{1}{2 \pi s \sin \left(\frac{3 \pi}{s}\right)}|u\rangle\langle v|
$$


has integral kernel

$$
\begin{aligned}
& u(x)\left(\frac{\eta(\varepsilon)-1}{(\mu \varepsilon)^{3-s}}-\frac{\eta_{s}}{\mu^{3-s}}\right) \mathrm{G}_{s,(\mu \varepsilon)^{s}}(x-y) v(y)+ \\
& \quad+u(x)\left(\frac{\mathrm{G}_{s,(\mu \varepsilon)^{s}}(x-y)-\mathrm{G}_{s, 0}(x-y)}{(\mu \varepsilon)^{3-s}}-\frac{1}{2 \pi s \sin \left(\frac{3 \pi}{s}\right)}\right) v(y) .
\end{aligned}
$$

The first summand in $\left(^{*}\right)$ vanishes as $\varepsilon \downarrow 0$ for a.e. $x, y \in \mathbb{R}^{3}$ as a consequence of Assumption $\left(\mathrm{I}_{s}\right)(\mathrm{ii})$, and so does the second summand in $(*)$ as a consequence of (4.11), where we take $\lambda=(\mu \varepsilon)^{s}$. Moreover, each such summand belongs to $L^{2}\left(\mathbb{R}^{3} \times\right.$ $\left.\mathbb{R}^{3}, \mathrm{~d} x \mathrm{~d} y\right)$ uniformly in $\varepsilon$, thanks to the assumption $\left(\mathrm{I}_{s}\right)(\mathrm{i})$ on the potentials $v$ and $u$. Thus, by dominated convergence, the function (*) vanishes in $L^{2}\left(\mathbb{R}^{3} \times \mathbb{R}^{3}, \mathrm{~d} x \mathrm{~d} y\right)$ as $\varepsilon \downarrow 0$, and this proves the limit (4.12) in the Hilbert-Schmidt norm.

We can now monitor the competing effect in $\varepsilon^{3-s}\left(\mathbb{1}+B_{\varepsilon}^{(s)}\right)^{-1}$ as $\varepsilon \downarrow 0$.

Lemma 4.6. Under the Assumptions $\left(\mathrm{I}_{s}\right)$ and $\left(\mathrm{II}_{s}\right)$ one has

$$
\lim _{\varepsilon \downarrow 0}(\mu \varepsilon)^{3-s}\left(\mathbb{1}+B_{\varepsilon}^{(s)}\right)^{-1}=\left(\frac{\eta_{s}}{\mu^{3-s}}+\frac{\left|\langle v, \phi\rangle_{L^{2}}\right|^{2}}{2 \pi s \sin \frac{3 \pi}{s}}\right)^{-1}|\phi\rangle\langle\widetilde{\phi}|
$$

in the operator norm topology.

Proof. We re-write (4.12) in the form of the expansion

$$
B_{\varepsilon}^{(s)}=B_{0}^{(s)}+(\mu \varepsilon)^{3-s} \mathcal{B}^{(s)}+o\left(\varepsilon^{3-s}\right)
$$

where, for short,

$$
\mathcal{B}^{(s)}:=\frac{\eta_{s}}{\mu^{3-s}} B_{0}^{(s)}+\frac{1}{2 \pi s \sin \left(\frac{3 \pi}{s}\right)}|u\rangle\langle v|,
$$

whence also

$$
\begin{aligned}
& (\mu \varepsilon)^{3-s}\left(\mathbb{1}+B_{\varepsilon}^{(s)}\right)^{-1}= \\
& =\left(\mathbb{1}+(\mu \varepsilon)^{3-s}\left(\mathbb{1}+(\mu \varepsilon)^{3-s}+B_{0}^{(s)}\right)^{-1}\left(\mathcal{B}^{(s)}-\mathbb{1}+o(1)\right)\right)^{-1} \times \\
& \quad \times(\mu \varepsilon)^{3-s}\left(\mathbb{1}+(\mu \varepsilon)^{3-s}+B_{0}^{(s)}\right)^{-1} .
\end{aligned}
$$

The $o\left(\varepsilon^{a}\right)$-remainders in (i) and (ii) above are clearly meant in the Hilbert-Schmidt norm. form

The operator $(\mu \varepsilon)^{3-s}\left(\mathbb{1}+(\mu \varepsilon)^{3-s}+B_{0}^{(s)}\right)^{-1}$ that appears twice in (ii) is of the

$$
z(\mathbb{1}+T+z \mathbb{1})^{-1}, \quad z \in \mathbb{C} \backslash\{0\},
$$

for a closed operator $T$ with isolated eigenvalue -1 ; this is a general setting for which a well-known expansion by Kato is available as $z \rightarrow 0$ [10, Sec. 3.6.5], which in the present context (in complete analogy with the argument of the proof of [3, Lemma I.1.2.4]) reads

$$
(\mu \varepsilon)^{3-s}\left(\mathbb{1}+(\mu \varepsilon)^{3-s}+B_{0}^{(s)}\right)^{-1}=-|\phi\rangle\langle\widetilde{\phi}|+O\left(\varepsilon^{3-s}\right)
$$

as $\varepsilon \downarrow 0$ in the operator norm topology. In practice, $\left(\mathbb{1}+(\mu \varepsilon)^{3-s}+B_{0}^{(s)}\right)^{-1}$ remains bounded also in the limit $\varepsilon \downarrow 0$ when restricted to the orthogonal complement of the eigenspace -1 of $B_{0}^{(s)}$, whereas it becomes singular when restricted to such eigenspace; the magnitude of the singularity is precisely $(\mu \varepsilon)^{-(3-s)}$, which is cancelled exactly by the pre-factor $(\mu \varepsilon)^{3-s}$ in the l.h.s. of (iii). In fact, by assumption of non-degeneracy, the eigenspace -1 is spanned by $\phi$ and $P:=-|\phi\rangle\langle\widetilde{\phi}|$ projects onto span $\{\phi\}$ with $P \phi=\phi$, as follows from the normalisation $\langle\widetilde{\phi}, \phi\rangle_{L^{2}}=-1$. 
Combining (ii) and (iii) above yields

$$
(\mu \varepsilon)^{3-s}\left(\mathbb{1}+B_{\varepsilon}^{(s)}\right)^{-1}=\left(\mathbb{1}+P\left(\mathcal{B}^{(s)}-\mathbb{1}\right)+O\left(\varepsilon^{3-s}\right)\right)^{-1}\left(P+O\left(\varepsilon^{3-s}\right)\right)
$$

as $\varepsilon \downarrow 0$ in the operator norm topology.

Next, in order to see that the limit $\varepsilon \downarrow 0$ in the r.h.s. of (iv) exists and is a bounded operator, we write explicitly

$$
\begin{aligned}
\mathbb{1}+P\left(\mathcal{B}^{(s)}-\mathbb{1}\right) & =\mathbb{1}-|\phi\rangle\langle\widetilde{\phi}|\left(\frac{\eta_{s}}{\mu^{3-s}} u(-\Delta)^{-\frac{s}{2}} v+\frac{1}{2 \pi s \sin \left(\frac{3 \pi}{s}\right)}|u\rangle\langle v|-\mathbb{1}\right) \\
& =\mathbb{1}+\frac{\eta_{s}}{\mu^{3-s}}|\phi\rangle\left\langle\widetilde{\phi}\left|-\frac{\overline{\langle v, \phi\rangle_{L^{2}}}}{2 \pi s \sin \left(\frac{3 \pi}{s}\right)}\right| \phi\right\rangle\langle v|+| \phi\rangle\langle\widetilde{\phi}|,
\end{aligned}
$$

where we used the identities $\langle\widetilde{\phi}, u\rangle_{L^{2}}=\langle\phi, v\rangle_{L^{2}}$ and

$$
\begin{aligned}
\left\langle\widetilde{\phi}, u(-\Delta)^{-\frac{s}{2}} v f\right\rangle_{L^{2}} & =\left\langle v(-\Delta)^{-\frac{s}{2}} u \widetilde{\phi}, f\right\rangle_{L^{2}}=\left\langle(\operatorname{sign} V) u(-\Delta)^{-\frac{s}{2}} v \phi, f\right\rangle_{L^{2}} \\
& =-\langle\widetilde{\phi}, f\rangle_{L^{2}} \quad \forall f \in L^{2}\left(\mathbb{R}^{3}\right) .
\end{aligned}
$$

Setting the constants

$$
\begin{aligned}
a & :=\left(\frac{\eta_{s}}{\mu^{3-s}}+1\right)\left(\frac{\eta_{s}}{\mu^{3-s}}+\frac{\left|\langle v, \phi\rangle_{L^{2}}\right|^{2}}{2 \pi s \sin \frac{3 \pi}{s}}\right)^{-1} \\
b & :=-\frac{\overline{\langle v, \phi\rangle_{L^{2}}}}{2 \pi s \sin \frac{3 \pi}{s}}\left(\frac{\eta_{s}}{\mu^{3-s}}+\frac{\left|\langle v, \phi\rangle_{L^{2}}\right|^{2}}{2 \pi s \sin \frac{3 \pi}{s}}\right)^{-1}
\end{aligned}
$$

the expression (v) allows one to compute explicitly (using again $\langle\widetilde{\phi}, \phi\rangle_{L^{2}}=-1$ )

$$
\left(\mathbb{1}+P\left(\mathcal{B}^{(s)}-\mathbb{1}\right)\right)(\mathbb{1}+a|\phi\rangle\langle\widetilde{\phi}|+b| \phi\rangle\langle v|)=\mathbb{1}
$$

and therefore to deduce that $\left(\mathbb{1}+P\left(\mathcal{B}^{(s)}-\mathbb{1}\right)\right)^{-1}$ exists and is bounded. This fact allows one to deduce from (iv) that

$$
\left.\lim _{\varepsilon \downarrow 0}(\mu \varepsilon)^{3-s}\left(\mathbb{1}+B_{\varepsilon}^{(s)}\right)^{-1}=\left(\mathbb{1}+P\left(\mathcal{B}^{(s)}-\mathbb{1}\right)\right)\right)^{-1} P
$$

in the operator norm topology.

Last, from (v), using $\langle\widetilde{\phi}, \phi\rangle_{L^{2}}=-1$ and $\langle\widetilde{\phi}, u\rangle_{L^{2}}=\langle\phi, v\rangle_{L^{2}}$, one finds

$$
\left(\mathbb{1}+P\left(\mathcal{B}^{(s)}-\mathbb{1}\right)\right) \phi=-\left(\frac{\eta_{s}}{\mu^{3-s}}+\frac{\left|\langle v, \phi\rangle_{L^{2}}\right|^{2}}{2 \pi s \sin \frac{3 \pi}{s}}\right) \phi
$$

and hence

$$
\left(\mathbb{1}+P\left(\mathcal{B}^{(s)}-\mathbb{1}\right)\right)^{-1} \phi=-\left(\frac{\eta_{s}}{\mu^{3-s}}+\frac{\left|\langle v, \phi\rangle_{L^{2}}\right|^{2}}{2 \pi s \sin \frac{3 \pi}{s}}\right)^{-1} \phi .
$$

Plugging the latter identity into (vi) yields finally (4.13) as a limit in the operator norm.

We are now in the condition to prove Theorem 2.3

Proof of Theorem 2.3. Owing to (4.7) we need to determine the limit of

$$
-A_{\varepsilon}^{(s)} \varepsilon^{3-s} \eta(\varepsilon)\left(\mathbb{1}+B_{\varepsilon}^{(s)}\right)^{-1} C_{\varepsilon}^{(s)}
$$

as $\varepsilon \downarrow 0$. As observed already, if $u(-\Delta)^{-\frac{s}{2}} v$ has no eigenvalue -1 , then the above expression vanishes with $\varepsilon$ and

$$
\left(h_{\varepsilon}^{(s / 2)}+\mu^{s} \mathbb{1}\right)^{-1} \stackrel{\varepsilon \downarrow 0}{\longrightarrow}\left((-\Delta)^{s / 2}+\mu^{s} \mathbb{1}\right)^{-1}
$$

in the operator norm. If instead $u(-\Delta)^{-\frac{s}{2}} v$ does admit a simple eigenvalue -1 , be $(-\Delta)^{\frac{s}{2}}+V$ zero-energy resonant or not, we are under the Assumption $\left(\mathrm{I}_{s}\right)$ and 
$\left(\mathrm{II}_{s}\right)$ of the present Section and we can therefore apply the limits (4.8), (4.10), and (4.13). This yields

$$
\begin{aligned}
& -A_{\varepsilon}^{(s)} \varepsilon^{3-s} \eta(\varepsilon)\left(\mathbb{1}+B_{\varepsilon}^{(s)}\right)^{-1} C_{\varepsilon}^{(s)} \\
& \stackrel{\varepsilon \downarrow 0}{\longrightarrow}-\left|\mathrm{G}_{s, \mu^{s}}\right\rangle\left\langle v\left|\circ\left(\eta_{s}+\frac{\mu^{3-s}\left|\langle v, \phi\rangle_{L^{2}}\right|^{2}}{2 \pi s \sin \frac{3 \pi}{s}}\right)^{-1}\right| \phi\right\rangle\langle\widetilde{\phi}|\circ| u\rangle\left\langle\mathrm{G}_{s, \mu^{s}}\right| \\
& \quad=-\frac{\left|\langle v, \phi\rangle_{L^{2}}\right|^{2}}{\eta_{s}+\frac{\mu^{3-s}\left|\langle v, \phi\rangle_{L^{2}}\right|^{2}}{2 \pi s \sin \frac{3 \pi}{s}}}\left|\mathrm{G}_{s, \mu^{s}}\right\rangle\left\langle\mathrm{G}_{s, \mu^{s}}\right|
\end{aligned}
$$

in the operator norm, having used $\langle\widetilde{\phi}, u\rangle_{L^{2}}=\langle\phi, v\rangle_{L^{2}}$. Now, if $(-\Delta)^{\frac{s}{2}}+V$ is not zero-energy resonant, then $\langle v, \phi\rangle_{L^{2}}=0$, owing to Theorem 2.2(iv), and the conclusion is again

$$
\left(h_{\varepsilon}^{(s / 2)}+\mu^{s} \mathbb{1}\right)^{-1} \stackrel{\varepsilon \downarrow 0}{\longrightarrow}\left((-\Delta)^{s / 2}+\mu^{s} \mathbb{1}\right)^{-1}
$$

in the operator norm. This proves part (i) of the present Theorem. If instead $(-\Delta)^{\frac{s}{2}}+V$ is zero-energy resonant, then using $\langle v, \phi\rangle_{L^{2}} \neq 0$ and plugging $\left(^{*}\right)$ back into (4.7) yields

$$
\begin{aligned}
\left(h_{\varepsilon}^{(s / 2)}+\mu^{s} \mathbb{1}\right)^{-1} \stackrel{\varepsilon \downarrow 0}{\longrightarrow}\left((-\Delta)^{s / 2}+\mu^{s} \mathbb{1}\right)^{-1} & \frac{1}{\frac{-\eta_{s}}{\left|\langle v, \phi\rangle_{L^{2}}\right|^{2}}-\frac{\mu^{3-s}}{2 \pi s \sin \frac{3 \pi}{s}}}\left|\mathrm{G}_{s, \mu^{s}}\right\rangle\left\langle\mathrm{G}_{s, \mu^{s}}\right|
\end{aligned}
$$

in the operator norm. Upon setting $\alpha:=-\eta_{s}\left|\langle v, \phi\rangle_{L^{2}}\right|^{-2}$ and $\lambda=\mu^{s}$, and comparing the resulting expression with (2.12), this means

$$
\begin{aligned}
\left(h_{\varepsilon}^{(s / 2)}+\lambda \mathbb{1}\right)^{-1} \stackrel{\varepsilon \downarrow 0}{\longrightarrow} & \left((-\Delta)^{s / 2}+\lambda \mathbb{1}\right)^{-1}+\left(\alpha-\frac{\lambda^{\frac{3}{s}-1}}{2 \pi s \sin \left(\frac{3 \pi}{s}\right)}\right)^{-1}\left|\mathrm{G}_{s, \lambda}\right\rangle\left\langle\mathrm{G}_{s, \lambda}\right| \\
& =\left(\mathrm{k}_{\alpha}^{(s / 2)}+\lambda \mathbb{1}\right)^{-1},
\end{aligned}
$$

which proves part (ii) of the Theorem.

\section{Convergence of the 1D limit: Resonant-Driven Case.}

The proof of the limit $h_{\varepsilon}^{(s / 2)} \stackrel{\varepsilon \downarrow 0}{\longrightarrow} \mathrm{k}_{\alpha}^{(s / 2)}$ in dimension one when $s \in\left(\frac{1}{2}, 1\right)$ (Theorem 3.3) is technically analogous to that in three dimensions. Therefore, based on the detailed discussion of the preceding Section, we only present here the steps of the convergence scheme and a sketch of their proofs.

Prior to that, let us set up the key resolvent identity and useful scaling properties with a notation that we can use also in Section 6 when we will deal with the resonant-independent limit.

We then keep $s \in\left(\frac{1}{2}, 1\right) \cup\left(1, \frac{3}{2}\right)$ generic for a moment and, in a unified form, we re-write (3.14) and (3.17) as

$$
V_{\varepsilon}(x)=\frac{\eta(\varepsilon)}{\varepsilon^{\frac{s+\gamma}{2}}} V\left(\frac{x}{\varepsilon}\right) .
$$

Taking $\gamma=s$ in (5.1) yields (3.14) and taking $\gamma=2-s$ yields (3.17). Thus, setting

$$
\begin{aligned}
v(x):=|V(x)|^{\frac{1}{2}}, \quad u(x) & :=|V(x)|^{\frac{1}{2}} \operatorname{sign}(V(x)), \\
v_{\varepsilon}(x) & :=\left|V_{\varepsilon}(x)\right|^{\frac{1}{2}}, \quad u_{\varepsilon}(x):=\left|V_{\varepsilon}(x)\right|^{\frac{1}{2}} \operatorname{sign}\left(V_{\varepsilon}(x)\right),
\end{aligned}
$$

one has

$$
v_{\varepsilon}(x)=\frac{\sqrt{\eta(\varepsilon)}}{\varepsilon^{(s+\gamma) / 4}} v\left(\frac{x}{\varepsilon}\right), \quad u_{\varepsilon}(x)=\frac{\sqrt{\eta(\varepsilon)}}{\varepsilon^{(s+\gamma) / 2}} u\left(\frac{x}{\varepsilon}\right), \quad v_{\varepsilon} u_{\varepsilon}=V_{\varepsilon} .
$$


The $1 \mathrm{D}$ analogue $U_{\varepsilon}: L^{2}(\mathbb{R}) \rightarrow L^{2}(\mathbb{R})$ of the unitary scaling operator (4.4) acts as

$$
\left(U_{\varepsilon} f\right)(x):=\frac{1}{\varepsilon^{1 / 2}} f\left(\frac{x}{\varepsilon}\right),
$$

which induces the scaling transformations

$$
\begin{aligned}
U_{\varepsilon}^{*} v_{\varepsilon} U_{\varepsilon} & =\frac{\sqrt{\eta(\varepsilon)}}{\varepsilon^{\frac{s+\gamma}{4}}} v, \quad U_{\varepsilon}^{*} u_{\varepsilon} U_{\varepsilon}=\frac{\sqrt{\eta(\varepsilon)}}{\varepsilon^{\frac{s+\gamma}{4}}} u, \\
U_{\varepsilon}^{*}\left((-\Delta)^{s / 2}+\lambda \mathbb{1}\right)^{-1} U_{\varepsilon} & =\varepsilon^{s}\left((-\Delta)^{s / 2}+\lambda \varepsilon^{s} \mathbb{1}\right)^{-1} .
\end{aligned}
$$

Based on arguments that differ depending on whether $s \in\left(\frac{1}{2}, 1\right)$ or $s \in\left(1, \frac{3}{2}\right)$ and which we shall prove in due time, the Konno-Kuroda-type resolvent identity

$$
\begin{aligned}
& \left(h_{\varepsilon}^{(s / 2)}+\lambda \mathbb{1}\right)^{-1}=\left((-\Delta)^{s / 2}+\lambda \mathbb{1}\right)^{-1}- \\
& -\left((-\Delta)^{s / 2}+\lambda \mathbb{1}\right)^{-1} v_{\varepsilon}\left(\mathbb{1}+u_{\varepsilon}\left((-\Delta)^{s / 2}+\lambda \mathbb{1}\right)^{-1} v_{\varepsilon}\right)^{-1} u_{\varepsilon}\left((-\Delta)^{s / 2}+\lambda \mathbb{1}\right)^{-1}
\end{aligned}
$$

holds as an identity between bounded operators on $L^{2}(\mathbb{R})$ for every $\varepsilon>0$ and every $-\lambda<0$ in the resolvent set of $h_{\varepsilon}^{(s / 2)}$. Inserting $U_{\varepsilon} U_{\varepsilon}^{*}=\mathbb{1}$ into (5.6) and applying (5.5) then yields

$$
\left(h_{\varepsilon}^{(s / 2)}+\lambda \mathbb{1}\right)^{-1}=\left((-\Delta)^{s / 2}+\lambda \mathbb{1}\right)^{-1}-A_{\varepsilon}^{(s)} \varepsilon^{\frac{2-s-\gamma}{2}} \eta(\varepsilon)\left(\mathbb{1}+B_{\varepsilon}^{(s)}\right)^{-1} C_{\varepsilon}^{(s)},
$$

having defined

$$
\begin{aligned}
A_{\varepsilon}^{(s)} & :=\varepsilon^{-\frac{2-s-\gamma}{2}}\left((-\Delta)^{s / 2}+\lambda \mathbb{1}\right)^{-1}(\eta(\varepsilon))^{-\frac{1}{2}} v_{\varepsilon} U_{\varepsilon} \\
B_{\varepsilon}^{(s)} & :=\eta(\varepsilon) \varepsilon^{\frac{s-\gamma}{2}} u\left((-\Delta)^{s / 2}+\lambda \varepsilon^{s} \mathbb{1}\right)^{-1} v \\
C_{\varepsilon}^{(s)} & :=U_{\varepsilon}^{*} u_{\varepsilon}(\eta(\varepsilon))^{-\frac{1}{2}}\left((-\Delta)^{s / 2}+\lambda \mathbb{1}\right)^{-1} \varepsilon^{-\frac{2-s-\gamma}{2}} .
\end{aligned}
$$

We shall see in a moment (Lemma 5.3) that $A_{\varepsilon}^{(s)}, B_{\varepsilon}^{(s)}$, and $C_{\varepsilon}^{(s)}$ are Hilbert-Schmidt operators on $L^{2}(\mathbb{R})$.

The following scaling property too is going to be useful in both regimes $s \in\left(\frac{1}{2}, 1\right)$ and $s \in\left(1, \frac{3}{2}\right)$.

Lemma 5.1. For any $s, \gamma, \varepsilon>0$ and any $x \in \mathbb{R} \backslash\{0\}$ one has

$$
\varepsilon^{\frac{s-\gamma}{2}} \mathrm{G}_{s, \lambda \varepsilon^{s}}(x)=\varepsilon^{\frac{2-s-\gamma}{2}} \mathrm{G}_{s, \lambda}(\varepsilon x) \text {. }
$$

Proof. Owing to (3.1)

$$
\begin{aligned}
\varepsilon^{\frac{s-\gamma}{2}} \mathrm{G}_{s, \lambda \varepsilon^{s}}(x) & =\frac{1}{2 \pi} \varepsilon^{\frac{s-\gamma}{2}} \int_{\mathbb{R}} \mathrm{d} p e^{\mathrm{i} p x} \frac{1}{|p|^{s}+\lambda \varepsilon^{s}} \\
& =\frac{1}{2 \pi} \varepsilon^{\frac{2-s-\gamma}{2}} \int_{\mathbb{R}} \mathrm{d} p e^{\mathrm{i} p(\varepsilon x)} \frac{1}{|p|^{s}+\lambda}=\varepsilon^{\frac{2-s-\gamma}{2}} \mathrm{G}_{s, \lambda}(\varepsilon x),
\end{aligned}
$$

whence the thesis.

We can now start the discussion for the proof of Theorem 3.3, thus working in the regime $s \in\left(\frac{1}{2}, 1\right)$.

First, we have the following properties.

Lemma 5.2. Let $V: \mathbb{R} \rightarrow \mathbb{R}$ belong to $L^{1}(\mathbb{R}) \cap \mathcal{R}_{s, 1}$ for some $s \in\left(\frac{1}{2}, 1\right)$. Then:

(i) for every $\lambda \geqslant 0,|V|^{\frac{1}{2}}\left((-\Delta)^{\frac{s}{2}}+\lambda \mathbb{1}\right)^{-1}|V|^{\frac{1}{2}}$ is a Hilbert-Schmidt operator on $L^{2}(\mathbb{R})$;

(ii) $|V|^{\frac{1}{2}} \ll(-\Delta)^{\frac{s}{4}}$ in the sense of infinitesimally bounded operators;

(iii) the operator $(-\Delta)^{\frac{s}{2}}+V$ defined as a form sum is self-adjoint and $\sigma_{\mathrm{ess}}\left((-\Delta)^{\frac{s}{2}}+\right.$ $V)=[0,+\infty)$. 
Proof. The proof is completely analogous to that of Lemma 4.1 for the 3D case, and is based on the fact that the integral kernel of $|V|^{\frac{1}{2}}\left((-\Delta)^{\frac{s}{2}}+\lambda \mathbb{1}\right)^{-1}|V|^{\frac{1}{2}}$, namely $|V(x)|^{\frac{1}{2}} \mathrm{G}_{s, \lambda}(x-y)|V(y)|^{\frac{1}{2}}$, belongs to $L^{2}(\mathbb{R} \times \mathbb{R}, \mathrm{d} x \mathrm{~d} y)$, as a direct consequence of the assumption $V \in L^{1}(\mathbb{R}) \cap \mathcal{R}_{s, 1}$.

Lemma 5.2 justifies the validity of the resolvent identity 5.60 , and hence of the rescaled identity (5.7), owing again to the general argument of [3, Theorem B.1(b)].

Next, we monitor separately the following limits.

Lemma 5.3. Let $V$ and $\eta$ satisfy Assumption $\left(\mathrm{I}_{s}^{-}\right)$for some $s \in\left(\frac{1}{2}, 1\right)$. For every $\varepsilon>0$, the operators $A_{\varepsilon}^{(s)}, B_{\varepsilon}^{(s)}$, and $C_{\varepsilon}^{(s)}$ defined by (5.1) (5.3) and (5.8) with $\gamma=s$ are Hilbert-Schmidt operators on $L^{2}(\mathbb{R})$ with limit

$$
\begin{aligned}
& \lim _{\varepsilon \downarrow 0} A_{\varepsilon}^{(s)}=\left|\mathrm{G}_{s, \lambda}\right\rangle\langle v| \\
& \lim _{\varepsilon \downarrow 0} B_{\varepsilon}^{(s)}=B_{0}^{(s)}=u(-\Delta)^{-\frac{s}{2}} v \\
& \lim _{\varepsilon \downarrow 0} C_{\varepsilon}^{(s)}=|u\rangle\left\langle\mathrm{G}_{s, \lambda}\right|
\end{aligned}
$$

in the Hilbert-Schmidt operator norm.

Proof. Completely analogous to the proof of Lemma 4.4 the integral kernels being now (with $\gamma=s$ )

$$
\begin{aligned}
& A_{\varepsilon}^{(s)}(x, y)=\mathrm{G}_{s, \lambda}(x-\varepsilon y) v(y) \\
& B_{\varepsilon}^{(s)}(x, y)=\eta(\varepsilon) u(x) \mathrm{G}_{s, \lambda \varepsilon^{s}}(x-y) v(y) \\
& C_{\varepsilon}^{(s)}(x, y)=u(x) \mathrm{G}_{s, \lambda}(\varepsilon x-y) .
\end{aligned}
$$

In particular, owing to (5.9),

$$
B_{\varepsilon}^{(s)}(x, y)=\eta(\varepsilon) \varepsilon^{1-s} u(x) \mathrm{G}_{s, \lambda}(\varepsilon x-\varepsilon y) v(y),
$$

and using (3.12)-3.13) one finds

$$
B_{\varepsilon}^{(s)}(x, y) \stackrel{\varepsilon \downarrow 0}{\longrightarrow} u(x) \frac{2^{1-s} \Gamma\left(\frac{1-s}{2}\right)}{(2 \pi)^{\frac{1}{2}} \Gamma\left(\frac{s}{2}\right)} \frac{1}{|x-y|^{1-s}} v(y)=B_{0}^{(s)}(x, y)
$$

pointwise almost everywhere.

Before plugging the limits found in Lemma (5.3) into (5.7), that now reads

$$
\left(h_{\varepsilon}^{(s / 2)}+\lambda \mathbb{1}\right)^{-1}=\left((-\Delta)^{s / 2}+\lambda \mathbb{1}\right)^{-1}-A_{\varepsilon}^{(s)} \varepsilon^{1-s} \eta(\varepsilon)\left(\mathbb{1}+B_{\varepsilon}^{(s)}\right)^{-1} C_{\varepsilon}^{(s)},
$$

we see that, since $B_{0}^{(s)}$ is compact, $\left(\mathbb{1}+B_{0}^{(s)}\right)^{-1}$ exists everywhere defined and bounded, in which case $\left(h_{\varepsilon}^{(s / 2)}+\lambda \mathbb{1}\right)^{-1} \rightarrow\left((-\Delta)^{s / 2}+\lambda \mathbb{1}\right)^{-1}$ as $\varepsilon \downarrow 0$, unless $B_{0}^{(s)}$ admits an eigenvalue -1 . We then consider the following additional assumption.

Assumption $\left(\mathbf{I I}_{s}^{-}\right)$. Assumption $\left(\mathrm{I}_{s}^{-}\right)$holds. $B_{0}^{(s)}$ has eigenvalue -1 , which is non-degenerate. $\phi \in L^{2}\left(\mathbb{R}^{3}\right)$ is a non-zero function such that $B_{0}^{(s)} \phi=-\phi$ and, in addition, $\langle\widetilde{\phi}, \phi\rangle_{L^{2}}=-1$, where $\widetilde{\phi}:=(\operatorname{sign} V) \phi$.

Since $\langle\widetilde{\phi}, \phi\rangle_{L^{2}}=-\left\langle(\operatorname{sign} V) \phi,(\operatorname{sign} V) v(-\Delta)^{-\frac{s}{2}} v \phi\right\rangle_{L^{2}}=-\left\|(-\Delta)^{-\frac{s}{4}} v \phi\right\|_{L^{2}}^{2}$, the normalisation $\langle\widetilde{\phi}, \phi\rangle_{L^{2}}=-1$ is always possible.

When Assumption $\left(\mathrm{II}_{s}^{-}\right)$holds, $\left(\mathbb{1}+B_{\varepsilon}^{(s)}\right)^{-1}$ becomes singular in the limit $\varepsilon \downarrow 0$, with a singularity that now competes with the vanishing factor $\varepsilon^{1-s}$ of (5.13). To resolve this competing effect, we need first to expand $B_{\varepsilon}^{(s)}$ around $\varepsilon=0$ to a further order, than the limit (5.11). This expansion is valid irrespectively of Assumption $\left(\mathrm{II}_{s}^{-}\right)$.

Lemma 5.4. Let $s \in\left(\frac{1}{2}, 1\right)$ and $\lambda>0$. 
(i) For every $x \in \mathbb{R} \backslash\{0\}$

$$
\lim _{\lambda \downarrow 0} \frac{\mathrm{G}_{s, \lambda}(x)-\mathrm{G}_{s, 0}(x)}{\left(s \sin \left(\frac{\pi}{s}\right)\right)^{-1} \lambda^{\frac{1}{s}-1}}=1 .
$$

(ii) In the norm operator topology one has

$$
\lim _{\varepsilon \downarrow 0} \frac{1}{\lambda^{\frac{1}{s}-1} \varepsilon^{1-s}}\left(B_{\varepsilon}^{(s)}-B_{0}^{(s)}\right)=\frac{\eta_{s}}{\lambda^{\frac{1}{s}-1}} B_{0}^{(s)}+\frac{1}{s \sin \left(\frac{3 \pi}{s}\right)}|u\rangle\langle v| .
$$

Here $\eta_{s} \in \mathbb{R}$ is the constant that is part of Assumption $\left(\mathrm{I}_{s}^{-}\right)$.

Proof. Completely analogous to the proof of Lemma 4.5 for the 3D case.

We can now monitor the competing effect in $\varepsilon^{1-s}\left(\mathbb{1}+B_{\varepsilon}^{(s)}\right)^{-1}$ as $\varepsilon \downarrow 0$.

Lemma 5.5. Under the Assumptions $\left(\mathrm{I}_{s}^{-}\right)$and $\left(\mathrm{II}_{s}^{-}\right)$one has

$$
\lim _{\varepsilon \downarrow 0} \varepsilon^{1-s}\left(\mathbb{1}+B_{\varepsilon}^{(s)}\right)^{-1}=\left(\eta_{s}+\frac{\left|\langle v, \phi\rangle_{L^{2}}\right|^{2}}{\lambda^{\frac{1}{s}-1} s \sin \frac{\pi}{s}}\right)^{-1}|\phi\rangle\langle\widetilde{\phi}|
$$

in the operator norm topology.

Proof. Completely analogous to the proof of Lemma 4.6 for the 3D case.

With these preliminaries at hand, we can prove Theorem 3.3

Proof of Theorem 3.3. The argument is the very same as the in the proof of Theorem 2.3 for the $3 \mathrm{D}$ case. Thus, the limit is the trivial one unless the potential in the approximating operators satisfy Assumptions $\left(\mathrm{I}_{s}^{-}\right)$and $\left(\mathrm{II}_{s}^{-}\right)$, in which case, plugging the limits (5.10), (5.12), and (5.16) into (5.13), one has

$$
\begin{aligned}
\left(h_{\varepsilon}^{(s / 2)}+\mu^{s} \mathbb{1}\right)^{-1} \stackrel{\varepsilon \downarrow 0}{\longrightarrow}\left((-\Delta)^{s / 2}+\mu^{s} \mathbb{1}\right)^{-1} & \\
& +\frac{1}{\frac{-\eta_{s}}{\left|\langle v, \phi\rangle_{L^{2}}\right|^{2}}-\frac{1}{\lambda^{1-\frac{1}{s}} s \sin \frac{3 \pi}{s}}}\left|\mathrm{G}_{s, \mu^{s}}\right\rangle\left\langle\mathrm{G}_{s, \mu^{s}}\right| .
\end{aligned}
$$

The comparison of the limit resolvent above with formulas (3.4) and (3.8) shows finally that the limit resolvent is precisely $\left(k_{\alpha}^{(s / 2)}+\lambda \mathbb{1}\right)^{-1}$ where the extension parameter satisfies $\alpha=-\eta_{s}\left|\int_{\mathbb{R}} \mathrm{d} x V(x) \psi(x)\right|^{-2}$, and this completes the proof.

\section{Convergence of the 1D limit: Resonant-independent CASE.}

This Section contains the proof of Theorem 3.4. Thus, now $s \in\left(1, \frac{3}{2}\right)$ and formulas (5.1)-(5.9) must be specialised with $\gamma=2-s$.

First, we observe that with $L^{1}$-potentials the following operator-theoretic properties hold.

Lemma 6.1. Let $V: \mathbb{R} \rightarrow \mathbb{R}$ belong to $L^{1}(\mathbb{R})$ and let $s \in\left(1, \frac{3}{2}\right)$. Then:

(i) for every $\lambda \geqslant 0,|V|^{\frac{1}{2}}\left((-\Delta)^{\frac{s}{2}}+\lambda \mathbb{1}\right)^{-1}|V|^{\frac{1}{2}}$ is a Hilbert-Schmidt operator on $L^{2}(\mathbb{R})$

(ii) $|V|^{\frac{1}{2}} \ll(-\Delta)^{\frac{s}{4}}$ in the sense of infinitesimally bounded operators;

(iii) the operator $(-\Delta)^{\frac{s}{2}}+V$ defined as a form sum is self-adjoint and $\sigma_{\text {ess }}\left((-\Delta)^{\frac{s}{2}}+\right.$ $V)=[0,+\infty)$.

Proof. Since $s>1$, (3.1) defines a function $\widehat{\mathrm{G}_{s, \lambda}} \in L^{1}(\mathbb{R})$, whence $\mathrm{G}_{s, \lambda} \in C_{\infty}(\mathbb{R})$ (continuous and vanishing at infinity). Therefore, the integral kernel of $|V|^{\frac{1}{2}}\left((-\Delta)^{\frac{s}{2}}+\right.$ $\lambda \mathbb{1})^{-1}|V|^{\frac{1}{2}}$, namely $|V(x)|^{\frac{1}{2}} \mathrm{G}_{s, \lambda}(x-y) \mid V(y)^{\frac{1}{2}}$, belongs to $L^{2}(\mathbb{R} \times \mathbb{R}, \mathrm{d} x \mathrm{~d} y)$, and this holds for any $\lambda \geqslant 0$. Based on this observation, the rest of the reasoning of the proof of Lemma 4.1 can be repeated verbatim. 
Following again the general argument of [3, Theorem B.1(b)], Lemma 6.1]justifies the validity of the resolvent identity (5.6), and hence of the rescaled identity (5.7), that now reads

$$
\left(h_{\varepsilon}^{(s / 2)}+\lambda \mathbb{1}\right)^{-1}=\left((-\Delta)^{s / 2}+\lambda \mathbb{1}\right)^{-1}-\eta(\varepsilon) A_{\varepsilon}^{(s)}\left(\mathbb{1}+B_{\varepsilon}^{(s)}\right)^{-1} C_{\varepsilon}^{(s)}
$$

for every $\varepsilon>0$ and every $-\lambda<0$ in the resolvent set of $h_{\varepsilon}^{(s / 2)}$.

Lemma 6.2. Let $V$ and $\eta$ satisfy Assumption $\left(\mathrm{I}_{s}^{+}\right)$for some $s \in\left(1, \frac{3}{2}\right)$. For every $\varepsilon>0$, the operators $A_{\varepsilon}^{(s)}, B_{\varepsilon}^{(s)}$, and $C_{\varepsilon}^{(s)}$ defined by (5.1) (5.3) and (5.8) with $\gamma=2-s$ are Hilbert-Schmidt operators on $L^{2}(\mathbb{R})$ with limit

$$
\begin{aligned}
& \lim _{\varepsilon \downarrow 0} A_{\varepsilon}^{(s)}=\left|\mathrm{G}_{s, \lambda}\right\rangle\langle v| \\
& \lim _{\varepsilon \downarrow 0} B_{\varepsilon}^{(s)}=B_{0}^{(s)}=\frac{\eta(0)}{\lambda^{1-\frac{1}{s}} s \sin \frac{\pi}{s}}|u\rangle\langle v| \\
& \lim _{\varepsilon \downarrow 0} C_{\varepsilon}^{(s)}=|u\rangle\left\langle\mathrm{G}_{s, \lambda}\right|
\end{aligned}
$$

in the Hilbert-Schmidt operator norm.

Proof. The integral kernels are now

$$
\begin{aligned}
A_{\varepsilon}^{(s)}(x, y) & =\mathrm{G}_{s, \lambda}(x-\varepsilon y) v(y) \\
B_{\varepsilon}^{(s)}(x, y) & =\eta(\varepsilon) \varepsilon^{s-1} u(x) \mathrm{G}_{s, \lambda \varepsilon^{s}}(x-y) v(y) \\
C_{\varepsilon}^{(s)}(x, y) & =u(x) \mathrm{G}_{s, \lambda}(\varepsilon x-y) .
\end{aligned}
$$

For $A_{\varepsilon}^{(s)}$ and $C_{\varepsilon}^{(s)}$ we reason precisely as in the proof of Lemma 4.4 $B_{\varepsilon}^{(s)}$ is HilbertSchmidt as a consequence of Lemma 6.1. Re-writing

$$
B_{\varepsilon}^{(s)}(x, y)=\eta(\varepsilon) u(x) \mathrm{G}_{s, \lambda}(\varepsilon x-\varepsilon y) v(y)
$$

by means of (5.9), and observing that (3.1) implies

$$
\mathrm{G}_{s, \lambda}(\varepsilon x-\varepsilon y) \stackrel{\varepsilon \downarrow 0}{\longrightarrow} \mathrm{G}_{s, \lambda}(0)=\frac{1}{\lambda^{1-\frac{1}{s}} s \sin \frac{\pi}{s}}
$$

one deduces

$$
B_{\varepsilon}^{(s)}(x, y) \stackrel{\varepsilon \downarrow 0}{\longrightarrow} \frac{\eta(0)}{\lambda^{1-\frac{1}{s}} s \sin \frac{\pi}{s}} u(x) v(y) .
$$

Then a dominated convergence argument, analogous to that used in the proof of Lemma 4.4 proves (6.3).

It is now convenient to observe the following (see [7, Lemma 5.1] for an analogous argument).

Lemma 6.3. Assume that the data $s \in\left(1, \frac{3}{2}\right), \lambda>0$ with $-\lambda$ in the resolvent set of all the $h_{\varepsilon}^{(s / 2)}$ 's, and $V$ and $\eta$ matching Assumption $\left(\mathrm{I}_{s}^{+}\right)$, do not satisfy the exceptional relation

$$
1+\frac{\eta(0)}{\lambda^{1-\frac{1}{s}} s \sin \frac{\pi}{s}} \int_{\mathbb{R}} \mathrm{d} x V(x)=0 .
$$

Then the operator $\mathbb{1}+B_{0}^{(s)}$ is invertible with bounded inverse, everywhere defined on $L^{2}(\mathbb{R})$. 
Proof. Since $B_{0}^{(s)}$ is compact on $L^{2}(\mathbb{R})$, based on the Fredholm alternative we have to prove that the validity of $(6.5)$ is equivalent to $B_{0}^{(s)}$ having eigenvalue -1 . In fact, $B_{0}^{(s)} \phi=-\phi$ for some non-zero $\phi \in L^{2}(\mathbb{R})$ is the same as

$$
\phi=\frac{\eta(0)}{\lambda^{1-\frac{1}{s}} s \sin \frac{\pi}{s}}\langle v, \phi\rangle_{L^{2}} u,
$$

meaning that $\phi$ is not orthogonal to $v$ and $\phi$ is a multiple of $u$. When this is the case, $u$ itself must be an eigenfunction of $B_{0}^{(s)}$ with eigenvalue -1 , and this is tantamount, owing to the identity above, as the validity of (6.5).

For given $s, \eta$, and $V$, the exceptional value of $-\lambda$ satisfying (6.5) is going to correspond to the negative eigenvalue of $\mathrm{k}_{\alpha}^{(s / 2)}$ described in Theorem [3.1)(iv). As we are going to monitor the limit $h_{\varepsilon}^{(s / 2)} \stackrel{\varepsilon \downarrow 0}{\longrightarrow} \mathrm{k}_{\alpha}^{(s / 2)}$ in the resolvent sense, not only must we discard the spectral points $-\lambda$ not belonging to the resolvent set of all the $h_{\varepsilon}^{(s / 2)}$ 's, but also the point $-\lambda$ given by (6.5). Thus, for our purposes the operator $\mathbb{1}+B_{0}^{(s)}$ is always invertible with everywhere defined bounded inverse.

In particular, 6.3) implies

$$
\left(\mathbb{1}+B_{\varepsilon}^{(s)}\right)^{-1} \stackrel{\varepsilon \downarrow 0}{\longrightarrow}\left(\mathbb{1}+B_{0}^{(s)}\right)^{-1}
$$

in the operator norm.

Based on the preceding preparatory materials, we can now prove Theorem 3.4

Proof of Theorem 3.4. Since (6.5) is excluded and therefore

$$
\left(\mathbb{1}+B_{0}^{(s)}\right)^{-1} u=\left(1+\frac{\eta(0) \int_{\mathbb{R}} \mathrm{d} x V(x)}{\lambda^{1-\frac{1}{s}} s \sin \frac{\pi}{s}}\right)^{-1} u,
$$

then plugging the limits (6.2), (6.4), and (6.6) into (6.1) yields

$$
\left(h_{\varepsilon}^{(s / 2)}+\lambda \mathbb{1}\right)^{-1} \stackrel{\varepsilon \downarrow 0}{\longrightarrow}\left((-\Delta)^{s / 2}+\lambda \mathbb{1}\right)^{-1}-\frac{\eta(0) \int_{\mathbb{R}} \mathrm{d} x V(x)}{1+\frac{\eta(0) \int_{\mathbb{R}} \mathrm{d} x V(x)}{\lambda^{1-\frac{1}{s}} s \sin \frac{\pi}{s}}}\left|\mathrm{G}_{s, \lambda}\right\rangle\left\langle\mathrm{G}_{s, \lambda}\right|
$$

in the operator norm. Upon setting

$$
\alpha:=-\left(\eta(0) \int_{\mathbb{R}} \mathrm{d} x V(x)\right)^{-1}
$$

and comparing the resulting expression with (3.4) and (3.8), one finds

$$
\begin{gathered}
\left(h_{\varepsilon}^{(s / 2)}+\lambda \mathbb{1}\right)^{-1} \stackrel{\varepsilon \downarrow 0}{\longrightarrow}\left((-\Delta)^{s / 2}+\lambda \mathbb{1}\right)^{-1}+\frac{1}{\alpha-\frac{1}{\lambda^{1-\frac{1}{s}} s \sin \frac{\pi}{s}}}\left|\mathrm{G}_{s, \lambda}\right\rangle\left\langle\mathrm{G}_{s, \lambda}\right| \\
=\left(\mathrm{k}_{\alpha}^{(s / 2)}+\lambda \mathbb{1}\right)^{-1},
\end{gathered}
$$

which completes the proof.

\section{ZERO-ENERGY RESONANCES FOR SCHRÖDINGER OPERATORS WITH FRACTIONAL LAPLACIAN}

The purpose of this Section is two-fold. First, we prove Theorems 2.2 and 3.2 . concerning the characterisation of the zero-energy resonant behaviour of $(-\Delta)^{s / 2}+$ $V$. Then, we discuss the occurrence of zero-energy resonances, both in one and three dimension. 
Proof of Theorem 2.2. The fact that for a real-valued $V \in L^{1}\left(\mathbb{R}^{3}\right) \cap \mathcal{R}_{s, 3}$ the operator $u(-\Delta)^{\frac{s}{2}} v$ is Hilbert-Schmidt follows from Lemma 4.1(i), thus part (i) is proved. Let us split

$$
\begin{aligned}
\psi(x) & =\left((-\Delta)^{-\frac{s}{2}} v \phi\right)(x)=\int_{\mathbb{R}^{3}} \mathrm{~d} y \frac{\Lambda_{s}}{|x-y|^{3-s}} v(y) \phi(y) \\
& =\frac{\Lambda_{s}\langle v, \phi\rangle_{L^{2}}}{|x|^{3-s}}+\Lambda_{s} \int_{\mathbb{R}^{3}} \mathrm{~d} y\left(\frac{1}{|x-y|^{3-s}}-\frac{1}{|x|^{3-s}}\right) v(y) \phi(y) \\
& \equiv \frac{\Lambda_{s}\langle v, \phi\rangle_{L^{2}}}{|x|^{3-s}}+\psi_{1}(x),
\end{aligned}
$$

where $\Lambda_{s}$ is the constant defined in (2.8). We now see that $\psi_{1} \in L^{2}\left(\mathbb{R}^{3}\right)$. To this aim, we observe that setting $\widehat{y}:=\frac{y}{|y|}$ one has

$$
\begin{aligned}
\int_{\mathbb{R}^{3}} \mathrm{~d} x & \left(\frac{1}{|x-y|^{3-s}}-\frac{1}{|x|^{3-s}}\right)^{2} \\
& =|y|^{2 s-3} \int_{\mathbb{R}^{3}} \mathrm{~d} x\left(\frac{|x-\widehat{y}|^{3-s}-|x|^{3-s}}{|x-\widehat{y}|^{3-s}|x|^{3-s}}\right)^{2} \\
& \lesssim|y|^{2 s-3} \int_{\mathbb{R}^{3}} \mathrm{~d} x\left(\frac{\langle x\rangle^{2-s}}{|x-\widehat{y}|^{3-s}|x|^{3-s}}\right)^{2}
\end{aligned}
$$

having used the change of variable $x \mapsto|y| x$ in the first step and the uniform bound ||$x-\left.\widehat{y}\right|^{3-s}-|x|^{3-s} \mid \lesssim\langle x\rangle^{2-s}$ in the last step. Since $s \in\left(\frac{3}{2}, \frac{5}{2}\right)$, the last integral above is finite, thus we deduce

$$
\int_{\mathbb{R}^{3}} \mathrm{~d} x\left(\frac{1}{|x-y|^{3-s}}-\frac{1}{|x|^{3-s}}\right)^{2} \lesssim|y|^{2 s-3} .
$$

As a consequence,

$$
\begin{aligned}
\left\|\psi_{1}\right\|_{L^{2}\left(\mathbb{R}^{3}\right)}^{2} & \lesssim \int_{\mathbb{R}^{3}} \mathrm{~d} x\left|\int_{\mathbb{R}^{3}} \mathrm{~d} y\left(\frac{1}{|x-y|^{3-s}}-\frac{1}{|x|^{3-s}}\right) v(y) \phi(y)\right|^{2} \\
& \leqslant \iint_{\mathbb{R}^{3} \times \mathbb{R}^{3}} \mathrm{~d} x \mathrm{~d} y\left(\frac{1}{|x-y|^{3-s}}-\frac{1}{|x|^{3-s}}\right)^{2}|V(y)| \\
& \lesssim \int_{\mathbb{R}^{3}} \mathrm{~d} y|V(y)||y|^{2 s-3}<+\infty
\end{aligned}
$$

as follows from a Cauchy-Schwartz inequality in the second step, from the bound (b) in the third step, and from the assumption $V \in L^{1}\left(\mathbb{R}^{3},\langle x\rangle^{2 s-3} \mathrm{~d} x\right)$ in the last step.

Since $|x|^{-(3-s)} \in L_{\text {loc }}^{2}\left(\mathbb{R}^{3}\right)$, because $s>\frac{3}{2}$, then identity (ait) implies that $\psi \in$ $L_{\text {loc }}^{2}\left(\mathbb{R}^{3}\right)$. Moreover, from (2.17) and (2.18) one finds

$$
V \psi=v u(-\Delta)^{-\frac{s}{2}} v \phi=-v \phi=-(-\Delta)^{\frac{s}{2}} \psi,
$$

whence $\left((-\Delta)^{\frac{s}{2}}+V\right) \psi=0$ distributionally. This completes the proof of part (ii).

Using (2.18) and the distributional identity proved in part (ii) one finds

$$
\langle v, \phi\rangle_{L^{2}}=\int_{\mathbb{R}^{3}} \mathrm{~d} x v(x) \phi(x)=\int_{\mathbb{R}^{3}} \mathrm{~d} x\left((-\Delta)^{\frac{s}{2}} \psi\right)(x)=-\int_{\mathbb{R}^{3}} \mathrm{~d} x V(x) \psi(x),
$$

which proves part (iii).

Last, the identity (aid) also implies that $\psi \in L^{2}\left(\mathbb{R}^{3}\right)$ is equivalent to $\langle v, \phi\rangle_{L^{2}}=0$. When this is the case, the identity $\left((-\Delta)^{\frac{s}{2}}+V\right) \psi=0$ holds in the $L^{2}$-sense, implying that $\psi \in \mathcal{D}\left((-\Delta)^{\frac{s}{2}}+V\right)$. This completes the proof of part (iv).

The proof of Theorem 3.2 proceeds along the same line. 
Proof of Theorem 3.2. Part (i) follows from Lemma $5.2(\mathrm{i})$.

Splitting

$$
\psi(x)=\left((-\Delta)^{-\frac{s}{2}} v \phi\right)(x)=\frac{\Lambda_{s}\langle v, \phi\rangle_{L^{2}}}{|x|^{1-s}}+\psi_{1}(x)
$$

where now $\Lambda_{s}$ is the constant defined in (3.13), and using the assumptions $V \in$ $L^{1}\left(\mathbb{R},\langle x\rangle^{2 s-1} \mathrm{~d} x\right)$ we can show that $\psi_{1} \in L^{2}(\mathbb{R})$, following the same argument as in the above proof of Theorem 2.2 . Therefore, since $|x|^{-(1-s)} \in L_{\text {loc }}^{2}(\mathbb{R})$ because $s>\frac{1}{2}$, from $\left(^{*}\right)$ one deduces that $\psi \in L_{\text {loc }}^{2}(\mathbb{R})$. Moreover, from (3.15) and (3.16) one finds $-V \psi=(-\Delta)^{\frac{s}{2}} \psi$, whence $\left((-\Delta)^{\frac{s}{2}}+V\right) \psi=0$ distributionally. Thus, part (ii) is proved.

Next, (3.16) and the distributional identity of part (ii) imply

$$
\langle v, \phi\rangle_{L^{2}}=-\int_{\mathbb{R}} \mathrm{d} x V(x) \psi(x),
$$

which proves part (iii).

Last, the identity $(*)$ also implies that $\psi \in L^{2}(\mathbb{R})$ is equivalent to $\langle v, \phi\rangle_{L^{2}}=0$, in which case $\left((-\Delta)^{\frac{s}{2}}+V\right) \psi=0$ in the $L^{2}$-sense, and this implies that $\psi \in$ $\mathcal{D}\left((-\Delta)^{\frac{s}{2}}+V\right)$. Thus, part (iv) is proved.

Let us address now the question of the existence of a potential $V$ such that the fractional Schrödinger operator $(-\Delta)^{s / 2}+V$ is zero-energy resonant.

Proposition 7.1. Let $\theta \in \mathcal{S}\left(\mathbb{R}^{3}\right)$, with $\theta>0$. Define

$$
\psi:=\theta * \mathrm{G}_{s, 0}=\theta * \frac{\Lambda_{s}}{|x|^{3-s}}, \quad V:=-\frac{\theta}{\psi},
$$

where $\Lambda_{s}$ is the constant defined in (2.8). Then $V$ satisfies part (i) of Assumption $\left(\mathrm{I}_{s}\right)$, and $(-\Delta)^{s / 2}+V$ is zero-energy resonant on $L^{2}\left(\mathbb{R}^{3}\right)$, with zero-energy resonance $\psi$.

Proof. By construction $\psi>0$, being the convolution of two strictly positive functions. Moreover, from

$$
\widehat{\psi}(p)=\frac{\widehat{\theta}(p)}{|p|^{s}}
$$

one sees that $\psi$ is continuous, as $\widehat{\psi} \in L^{1}\left(\mathbb{R}^{3}\right)$, and that $\psi \notin L^{2}\left(\mathbb{R}^{3}\right)$, as $\widehat{\psi} \notin L^{2}\left(\mathbb{R}^{3}\right)$ either. Still, for every compact $K \subset \mathbb{R}^{3}$

$$
\left\|\left(\theta * \mathrm{G}_{s, 0}\right) \mathbf{1}_{K}\right\|_{L^{2}} \leqslant\|\theta\|_{L^{\frac{6}{5}}}\left\|\mathrm{G}_{s, 0}\right\|_{L^{2}}\left\|\mathbf{1}_{K}\right\|_{L^{6}}<+\infty
$$

as follows by means of a Schwartz and a Young inequality. Thus,

$$
\psi \in L_{\mathrm{loc}}^{2}\left(\mathbb{R}^{3}\right) \backslash L^{2}\left(\mathbb{R}^{3}\right) .
$$

Next, we argue that the leading decay in $\psi$ is $|x|^{-(3-s)}$. To see that, since $\theta(x) \lesssim\langle x\rangle^{-m}$ for any $m \in \mathbb{N}$, we write

$$
\begin{aligned}
\psi(x) & \lesssim \int_{\mathbb{R}^{3}} \frac{1}{\langle x-y\rangle^{m}} \frac{1}{|y|^{3-s}} \mathrm{~d} y \\
& =\int_{|y-x| \geqslant \frac{1}{2}|x|} \frac{1}{\langle y-x\rangle^{m}} \frac{1}{|y|^{3-s}} \mathrm{~d} y+\int_{|y-x|<\frac{1}{2}|x|} \frac{1}{\langle y-x\rangle^{m}} \frac{1}{|y|^{3-s}} \mathrm{~d} y
\end{aligned}
$$


and we take non-restrictively $|x| \geqslant 1$ and $m \geqslant 4$. The first integral in the r.h.s. of (ii) is estimated as

$$
\begin{aligned}
& \int_{|y-x| \geqslant \frac{1}{2}|x|} \frac{1}{\langle y-x\rangle^{m}} \frac{1}{|y|^{3-s}} \mathrm{~d} y \\
& \quad=\int_{\substack{|y-x| \geqslant \frac{1}{2}|x| \\
|y| \geqslant \frac{3}{2}|x| \geqslant \frac{3}{2}}} \frac{1}{\langle y-x\rangle^{m}} \frac{1}{|y|^{3-s}} \mathrm{~d} y+\int_{\substack{|y-x| \geqslant \frac{1}{2}|x| \\
|y|<\frac{3}{2}|x|}} \frac{1}{\langle y-x\rangle^{m}} \frac{1}{|y|^{3-s}} \mathrm{~d} y \\
& \quad \lesssim \frac{1}{|x|^{m-4}} \int_{\mathbb{R}^{3}} \frac{\mathrm{d} y}{\langle y-x\rangle^{4}}+\frac{1}{|x|^{m}} \int_{|y|<\frac{3}{2}|x|} \frac{\mathrm{d} y}{|y|^{3-s}} \lesssim \frac{1}{|x|^{m-4}}+\frac{1}{|x|^{m-s}}
\end{aligned}
$$

and hence vanishes as $|x| \rightarrow+\infty$ faster than any power. Concerning the second integral in the r.h.s. of (ii), since $|y-x|<\frac{1}{2}|x|$ implies $|y|>\frac{1}{2}|x|$, one has

$$
\int_{|y-x|<\frac{1}{2}|x|} \frac{1}{\langle y-x\rangle^{m}} \frac{1}{|y|^{3-s}} \mathrm{~d} y \lesssim \frac{1}{|x|^{3-s}} \int_{\mathbb{R}^{3}} \frac{\mathrm{d} y}{\langle y-x\rangle^{m}} \lesssim \frac{1}{|x|^{3-s}} .
$$

Therefore, (ii) implies

$$
\psi(x) \lesssim\langle x\rangle^{-(3-s)}
$$

Now, splitting

$$
\psi=\frac{\Lambda_{s} \widehat{\theta}(0)}{|x|^{3-s}}+\psi_{1}, \quad \psi_{1}=\left(\frac{\widehat{\theta}(\cdot)-\widehat{\theta}(0)}{|\cdot|^{s}}\right)^{\vee}
$$

and observing that $\widehat{\psi_{1}} \in L^{2}\left(\mathbb{R}^{3}\right)$ (owing to the bound $|\widehat{\theta}(p)-\widehat{\theta}(0)| \lesssim|p|$, valid for small $|p|)$, whence also $\psi_{1} \in L^{2}\left(\mathbb{R}^{3}\right)$, we deduce that in order for the decay $\psi_{1}(x) \lesssim\langle x\rangle^{-(3-s)}$ (whose bound is not square-integrable) implied by (iii) above to be compatible with the square-integrability of the continuous function $\psi_{1}, \psi_{1}$ itself must decay more that $\langle x\rangle^{-(3-s)}$, which allows us to conclude that the leading decay in $\psi$ is $|x|^{-(3-s)}$.

Since $\psi$ is continuous, positive, and with polynomial decay $|x|^{-(3-s)}$ at infinity, then $1 / \psi$ is continuous, positive, and with polynomial growth at infinity. Since $\theta$ is a Schwartz function, we conclude that $V=\theta \frac{1}{\psi}$ is continuous and decays at infinity faster than any polynomial.

As a consequence, $V \in L^{1}\left(\mathbb{R}^{3},\langle x\rangle^{2 s-3} \mathrm{~d} x\right)$ and also $\|V\|_{\mathcal{R}_{s, 3}} \lesssim\|V\|_{L^{3 / s}}<+\infty$. This proves that $V$ satisfies part (i) of Assumption $\left(\mathrm{I}_{s}\right)$.

By construction, $(-\Delta)^{s / 2} \psi=\theta$, whence

$$
\left((-\Delta)^{s / 2}+V\right) \psi=0
$$

distributionally. This also implies

$$
(-\Delta)^{-s / 2} V \psi=-\psi
$$

Writing as usual $V=u v$ with $v=|V|^{\frac{1}{2}}$ and $u=|V|^{\frac{1}{2}} \operatorname{sign}(V)$, let us now set $\phi:=-u \psi$. Then (v) yields

$$
\psi=(-\Delta)^{-\frac{s}{2}} v \phi,
$$

which is precisely the relation between $\psi$ and $\phi$ given by (2.18). $\phi$ cannot be identically zero, because so would be $\psi$, owing to (vi), which is not the case. Moreover, $\phi$ is square-integrable, because in the product $u \psi$ the $L_{\text {loc }}^{2}$-function $\psi$ is multiplied by a function that decays more than polynomially at infinity. Therefore,

$$
\phi \in L^{2}\left(\mathbb{R}^{3}\right) \backslash\{0\},
$$

and, multiplying (v) by $u$,

$$
u(-\Delta)^{-\frac{s}{2}} v \phi=-\phi
$$

as an identity in $L^{2}\left(\mathbb{R}^{3}\right)$. 
Conditions (i), (iv), (vi), (vii), and (viii) above, owing to Theorem 2.2 and to the present definition of resonance, imply that $(-\Delta)^{s / 2}+V$ is zero-energy resonant, with zero-energy resonance $\psi$.

The counterpart result in $1 \mathrm{D}$, which we state here below, has a completely analogous proof, that we then omit.

Proposition 7.2. Let $\theta \in \mathcal{S}(\mathbb{R})$, with $\theta>0$. Define

$$
\psi:=\theta * \mathrm{G}_{s, 0}=\theta * \frac{\Lambda_{s}}{|x|^{1-s}}, \quad V:=-\frac{\theta}{\psi},
$$

where $\Lambda_{s}$ is the constant defined in (3.13) Then $V$ satisfies part (i) of Assumption $\left(\mathrm{I}_{s}^{-}\right)$, and $(-\Delta)^{s / 2}+V$ is zero-energy resonant on $L^{2}(\mathbb{R})$, with zero-energy resonance $\psi$.

Remark 7.3. By means of a more refined discussion, in the same spirit of [11, we can identify the threshold coupling parameter $\lambda \in \mathbb{R}$, for a given potential $V$ in a suitable class, for which $(-\Delta)^{s / 2}+\lambda V$ is zero-energy resonant. We do not develop this interesting approach here.

\section{REFERENCES}

[1] S. Albeverio, F. Gesztesy, R. Hø egh-Krohn, And H. Holden, Point interactions in two dimensions: basic properties, approximations and applications to solid state physics, J. Reine Angew. Math., 380 (1987), pp. 87-107.

[2] S. Albeverio, F. Gesztesy, and R. Høegh-Krohn, The low energy expansion in nonrelativistic scattering theory, Ann. Inst. H. Poincaré Sect. A (N.S.), 37 (1982), pp. 1-28.

[3] S. Albeverio, F. Gesztesy, R. Høegh-Krohn, and H. Holden, Solvable Models in Quantum Mechanics, Texts and Monographs in Physics, Springer-Verlag, New York, 1988.

[4] S. Albeverio, F. Gesztesy, R. Høegh-Krohn, and W. Kirsch, On point interactions in one dimension, J. Operator Theory, 12 (1984), pp. 101-126.

[5] S. Albeverio And P. Kurasov, Singular perturbations of differential operators, vol. 271 of London Mathematical Society Lecture Note Series, Cambridge University Press, Cambridge, 2000. Solvable Schrödinger type operators.

[6] E. Capelas de Oliveira and J. J. Vaz, Tunneling in fractional quantum mechanics, J. Phys. A, 44 (2011), pp. 185303, 17.

[7] G. Dell'Antonio And A. Michelangeli, Schrödinger operators on half-line with shrinking potentials at the origin, Asymptot. Anal., 97 (2016), pp. 113-138.

[8] M. Gallone, A. Michelangeli, and A. Ottolini, Kreĭn-Višik-Birman self-adjoint extension theory revisited, SISSA preprint 25/2017/MATE (2017).

[9] S. Jarosz and J. J. VAz, Fractional Schrödinger equation with Riesz-Feller derivative for delta potentials, J. Math. Phys., 57 (2016), pp. 123506, 16.

[10] T. KATo, Perturbation theory for linear operators, Classics in Mathematics, Springer-Verlag, Berlin, 1995. Reprint of the 1980 edition.

[11] M. Klaus and B. Simon, Coupling constant thresholds in nonrelativistic quantum mechanics. I. Short-range two-body case, Ann. Physics, 130 (1980), pp. 251-281.

[12] R. Konno and S. T. Kuroda, On the finiteness of perturbed eigenvalues, J. Fac. Sci. Univ. Tokyo Sect. I, 13 (1966), pp. 55-63 (1966).

[13] E. K. Lenzi, H. V. Ribeiro, M. A. F. dos Santos, R. Rossato, and R. S. Mendes, Time dependent solutions for a fractional Schrödinger equation with delta potentials, J. Math. Phys., 54 (2013), pp. 082107, 8.

[14] A. Michelangeli, A. Ottolini, and R. Scandone, Fractional powers and singular perturbations of differential operators, arXiv:1801.08885 (2018).

[15] S. I. Muslih, Solutions of a particle with fractional $\delta$-potential in a fractional dimensional space, Internat. J. Theoret. Phys., 49 (2010), pp. 2095-2104.

[16] M. M. NAYGa AND J. P. EsguerRA, Green's functions and energy eigenvalues for deltaperturbed space-fractional quantum systems, J. Math. Phys., 57 (2016), pp. 022103, 7.

[17] E. C. D. Oliveira, F. S. Costa, And J. J. VAz, The fractional Schrödinger equation for delta potentials, J. Math. Phys., 51 (2010), pp. 123517, 16.

[18] A. SACChetTI, Stationary solutions of a fractional Laplacian with singular perturbation, arXiv:1801.01694 (2018). 
POINT-LIKE PERTURBED FRACTIONAL LAPLACIANS WITH SHRINKING POTENTIALS27

[19] T. Sandev, I. Petreska, And E. K. Lenzi, Time-dependent Schrödinger-like equation with nonlocal term, J. Math. Phys., 55 (2014), pp. 092105, 10.

[20] B. Simon, Trace ideals and their applications, vol. 120 of Mathematical Surveys and Monographs, American Mathematical Society, Providence, RI, second ed., 2005.

[21] J. D. TARe And J. P. H. Esguerra, Bound states for multiple Dirac- $\delta$ wells in spacefractional quantum mechanics, J. Math. Phys., 55 (2014), pp. 012106, 10.

(A. Michelangeli) International School for Advanced Studies - SiSSA, via Bonomea 265, 34136 Trieste (ItALY).

E-mail address: alemiche@sissa.it

(R. Scandone) International School for Advanced Studies - SiSSA, via Bonomea 265, 34136 Trieste (ItAly).

E-mail address: rscandone@sissa.it 